\title{
Multi-indexed (q-)Racah Polynomials
}

\author{
Satoru Odake ${ }^{a}$ and Ryu Sasaki ${ }^{b}$ \\ ${ }^{a}$ Department of Physics, Shinshu University, \\ Matsumoto 390-8621, Japan \\ ${ }^{b}$ Yukawa Institute for Theoretical Physics, \\ Kyoto University, Kyoto 606-8502, Japan
}

\begin{abstract}
As the second stage of the project multi-indexed orthogonal polynomials, we present, in the framework of 'discrete quantum mechanics' with real shifts in one dimension, the multi-indexed $(q-)$ Racah polynomials. They are obtained from the $(q-)$ Racah polynomials by multiple application of the discrete analogue of the Darboux transformations or the Crum-Krein-Adler deletion of 'virtual state' vectors, in a similar way to the multi-indexed Laguerre and Jacobi polynomials reported earlier. The virtual state vectors are the 'solutions' of the matrix Schrödinger equation with negative 'eigenvalues', except for one of the two boundary points.
\end{abstract}

\section{Introduction}

This is a second report of the project multi-indexed orthogonal polynomials. Following the examples of multi-indexed Laguerre and Jacobi polynomials [1, multi-indexed (q-)Racah polynomials are constructed in the framework of discrete quantum mechanics with real shifts [2]. It should be emphasised that the original $(q-)$ Racah polynomials are the most generic members of the Askey scheme of hypergeometric orthogonal polynomials with purely discrete orthogonality measures [3, 4, 5, 6]. They are also called orthogonal polynomials of a discrete variable [7]. These new multi-indexed orthogonal polynomials are specified by a set of indices $\mathcal{D}=\left\{d_{1}, \ldots, d_{M}\right\}$ consisting of distinct natural numbers $d_{j} \in \mathbb{N}$, on top of $n$, which counts the nodes as in the ordinary orthogonal polynomials. The simplest examples, $\mathcal{D}=\{\ell\}, \ell \geq 1$, $\left\{P_{\ell, n}(x)\right\}$ are also called exceptional orthogonal polynomials [8]-[27]. They are obtained as the 
main part of the eigenfunctions (vectors) of various exactly solvable Schrödinger equations in one dimensional quantum mechanics and their 'discrete' generalisations, in which the corresponding Schrödinger equations are second order difference equations [2, 28, 29]. They form a complete set of orthogonal polynomials, although they start at a certain positive degree $(\ell \geq 1)$ rather than a degree zero constant term. The latter situation is essential for avoiding the constraints of Bochner's theorem [30]. The exceptional Laguerre polynomials with two extra indices $\mathcal{D}=\left\{d_{1}, d_{2}\right\}$ were introduced in [31]. We are quite sure that these new orthogonal polynomials will find plenty of novel applications in various branches of science and technology as other orthogonal polynomials. One obvious application is the birth and death processes [32]. These new orthogonal polynomials provide huge stocks of exactly solvable birth and death processes [33. The transition probabilities are given explicitly, not in a general spectral representation form of Karlin-McGregor [34. An interesting possible application is to one-dimensional spin systems and quantum information theory [35].

The basic logic for constructing multi-indexed orthogonal polynomials is essentially the same for the ordinary Schrödinger equations, i.e. those for the Laguerre and Jacobi polynomials and for the difference Schrödinger equations with real shifts, i.e. the (q-)Racah polynomials, etc. The main ingredients are the factorised Hamiltonians, the Crum-Krein-Adler formulas [36, 37, 38] for deletion of eigenstates, that is the multiple Darboux transformations [39] and the virtual states solutions [1] which are generated by twisting the discrete symmetries of the original Hamiltonians. Most of these methods for discrete Schrödinger equations had been developed [2, 26, 28, 29, 40, 41, 42] and they were used for the exceptional $(q-)$ Racah polynomials [23]. The concept of virtual state 'solutions' requires special explanation in the present case. In the ordinary quantum mechanics cases, the virtual state solutions are the solutions of the Schrödinger equation but they do not belong to the Hilbert space of square integrable functions due to the twisted boundary conditions. In the present case, the Hamiltonians are finite-dimensional real symmetric tri-diagonal matrices. Therefore the eigenvalue equations for a given Hamiltonian matrix cannot have any extra solutions other than the genuine eigenvectors. Thus we will use the term virtual state vectors. As will be shown in the text, virtual state vectors are the 'solutions' of the eigenvalue problem for a virtual Hamiltonian $\mathcal{H}^{\prime}$, except for one of the boundaries, $x=x_{\max }(2.50)$. The virtual Hamiltonians are obtained from the original Hamiltonian by twisting the discrete symmetry and they are linearly related to the original Hamiltonian (2.46). Thus the virtual state vectors 'satisfy' 
the eigenvalue equation for the original Hamiltonian, except for one of the two boundaries. The polynomial part of the virtual state vectors had been used for the exceptional $(q-)$ Racah polynomials. One distinctive feature of virtual states deletion in discrete quantum mechanics with real shifts is that the size of the Hamiltonian matrix $\left(x_{\max }\right)$ remains the same. This is in marked contrast with the eigenstates deletion (Christoffel transformations [3, 42]), in which case the size decreases by the number of deleted eigenstates.

This paper is organised as follows. In section two, the main ingredients of the theory, the difference Schrödinger equation for the $(q-)$ Racah system, the polynomial eigenvectors and virtual state vectors, are introduced. Starting from the general setting of discrete quantum mechanics with real shifts in $\S 2.1$, the basic properties of the $(q-)$ Racah systems are recapitulated in $\S 2.2$. Based on the twisting (symmetry), the virtual state vectors are introduced in $\S 2.3$. Section three is the main part of the paper. The basic logic of virtual states deletion in discrete quantum mechanics with real shifts in general is outlined in $\S 3.1$. The explicit forms of multi-indexed $(q-)$ Racah polynomials are provided in $\oint \underline{3.2}$. The final section is for a summary and comments.

\section{Original System}

\subsection{General formulation}

Let us recapitulate the discrete quantum mechanics with real shifts developed in [2]. We restrict ourselves to the finite dimensional matrix case, $x_{\max }=N$.

The Hamiltonian $\mathcal{H}=\left(\mathcal{H}_{x, y}\right)$ is an irreducible (that is, not the direct sum of two or more such matrices) tri-diagonal real symmetric (Jacobi) matrix and its rows and columns are indexed by non-negative integers $x$ and $y, x, y=0,1, \ldots, x_{\max }$. By adding a scalar matrix to the Hamiltonian, the lowest eigenvalue is assumed to be zero. This makes the Hamiltonian positive semi-definite. By a similarity transformation in terms of a diagonal matrix of \pm 1 entries only, the eigenvector corresponding to the zero eigenvalue can be made to have definite sign, i.e. all the components are positive or negative. Then the Hamiltonian $\mathcal{H}$ has the following form

$$
\mathcal{H}_{x, y} \stackrel{\text { def }}{=}-\sqrt{B(x) D(x+1)} \delta_{x+1, y}-\sqrt{B(x-1) D(x)} \delta_{x-1, y}+(B(x)+D(x)) \delta_{x, y}
$$

in which the potential functions $B(x)$ and $D(x)$ are real and positive but vanish at the 
boundary:

$$
\begin{aligned}
& B(x)>0 \quad\left(x=0,1, \ldots, x_{\max }-1\right), \quad B\left(x_{\max }\right)=0, \\
& D(x)>0 \quad\left(x=1,2, \ldots, x_{\max }\right), \quad D(0)=0 .
\end{aligned}
$$

The Schrödinger equation is the eigenvalue problem for the hermitian matrix $\mathcal{H}$,

$$
\mathcal{H} \phi_{n}(x)=\mathcal{E}_{n} \phi_{n}(x) \quad\left(n=0,1, \ldots, n_{\max }\right), \quad 0=\mathcal{E}_{0}<\mathcal{E}_{1}<\cdots<\mathcal{E}_{n_{\max }},
$$

where the eigenvector is $\phi_{n}=\left(\phi_{n}(x)\right)_{x=0,1, \ldots, x_{\max }}$ and $n_{\max }=N$. Reflecting the positive semi-definiteness and based on the boundary conditions (2.2), the Hamiltonian (2.1) can be expressed in a factorised form:

$$
\begin{aligned}
& \mathcal{H}=\mathcal{A}^{\dagger} \mathcal{A}, \quad \mathcal{A}=\left(\mathcal{A}_{x, y}\right), \quad \mathcal{A}^{\dagger}=\left(\left(\mathcal{A}^{\dagger}\right)_{x, y}\right)=\left(\mathcal{A}_{y, x}\right), \quad\left(x, y=0,1, \ldots, x_{\max }\right), \\
& \mathcal{A}_{x, y} \stackrel{\text { def }}{=} \sqrt{B(x)} \delta_{x, y}-\sqrt{D(x+1)} \delta_{x+1, y}, \quad\left(\mathcal{A}^{\dagger}\right)_{x, y}=\sqrt{B(x)} \delta_{x, y}-\sqrt{D(x)} \delta_{x-1, y} .
\end{aligned}
$$

Here $\mathcal{A}\left(\mathcal{A}^{\dagger}\right)$ is an upper (lower) triangular matrix with the diagonal and the super(sub)diagonal entries only. The zero mode equation, $\mathcal{A} \phi_{0}=0$, is

$$
\begin{aligned}
& \sqrt{B(x)} \phi_{0}(x)-\sqrt{D(x+1)} \phi_{0}(x+1)=0 \quad\left(x=0,1, \ldots, x_{\max }-1\right), \\
& \sqrt{B\left(x_{\max }\right)} \phi_{0}\left(x_{\max }\right)=0,
\end{aligned}
$$

and the second equation is trivially satisfied by the boundary condition $B\left(x_{\max }\right)=0$. The groundstate eigenvector is easily obtained:

$$
\phi_{0}(x)=\sqrt{\prod_{y=0}^{x-1} \frac{B(y)}{D(y+1)}}\left(x=0,1, \ldots, x_{\max }\right),
$$

with the normalisation $\phi_{0}(0)=1$ (convention: $\prod_{k=n}^{n-1} *=1$ ). Needless to say it is positive for $x=0,1, \ldots, x_{\max }$. For the explicit examples treated in [2], $\phi_{0}^{2}(x)$ can be analytically continued to the entire complex $x$-plane as a meromorphic function and it vanishes on the integer points outside the boundary; $\phi_{0}^{2}(x)=0\left(x \in \mathbb{Z} \backslash\left\{0,1, \ldots, x_{\max }\right\}\right)$. The eigenvectors are mutually orthogonal:

$$
\left(\phi_{n}, \phi_{m}\right) \stackrel{\text { def }}{=} \sum_{x=0}^{x_{\max }} \phi_{n}(x) \phi_{m}(x)=\frac{1}{d_{n}^{2}} \delta_{n m} \quad\left(n, m=0,1, \ldots, n_{\max }\right) .
$$

For simplicity in notation, we write $\mathcal{H}, \mathcal{A}$ and $\mathcal{A}^{\dagger}$ as follows:

$$
e^{ \pm \partial}=\left(\left(e^{ \pm \partial}\right)_{x, y}\right) \quad\left(x, y=0,1, \ldots, x_{\max }\right), \quad\left(e^{ \pm \partial}\right)_{x, y} \stackrel{\text { def }}{=} \delta_{x \pm 1, y}, \quad\left(e^{\partial}\right)^{\dagger}=e^{-\partial},
$$




$$
\begin{aligned}
\mathcal{H} & =-\sqrt{B(x)} e^{\partial} \sqrt{D(x)}-\sqrt{D(x)} e^{-\partial} \sqrt{B(x)}+B(x)+D(x) \\
& =-\sqrt{B(x) D(x+1)} e^{\partial}-\sqrt{B(x-1) D(x)} e^{-\partial}+B(x)+D(x), \\
\mathcal{A} & =\sqrt{B(x)}-e^{\partial} \sqrt{D(x)}, \quad \mathcal{A}^{\dagger}=\sqrt{B(x)}-\sqrt{D(x)} e^{-\partial} .
\end{aligned}
$$

For the Schrödinger equation (2.22), it is sufficient that the functions $B(x), D(x)$ and $\phi_{n}(x)$ are defined only for the integer grid, $x=0,1, \ldots, x_{\max }$. In this paper we consider the case that the potential functions $B(x)$ and $D(x)$ are rational functions of $x$ or $q^{x}(0<q<1)$. So they are defined for any $x \in \mathbb{C}$ (except for the zeros of their denominators), see the explicit forms (2.20)-(2.21). Also we consider the eigenvectors in a factorised form:

$$
\phi_{n}(x)=\phi_{0}(x) \check{P}_{n}(x), \quad \check{P}_{n}(x) \stackrel{\text { def }}{=} P_{n}(\eta(x))
$$

Here $P_{n}(\eta)$ is a polynomial of degree $n$ in $\eta$ and the sinusoidal coordinate $\eta(x)$ is one of the following [2]; $\eta(x)=x, \epsilon^{\prime} x(x+d), 1-q^{x}, q^{-x}-1, \epsilon^{\prime}\left(q^{-x}-1\right)\left(1-d q^{x}\right),\left(\epsilon^{\prime}= \pm 1\right)$. Since $P_{n}$ is a polynomial, $\check{P}_{n}(x)$ is defined for any $x \in \mathbb{C}$. The Schrödinger equation (2.22) gives a square root free difference equation for the polynomial eigenvector $\check{P}_{n}(x)$,

$$
B(x)\left(\check{P}_{n}(x)-\check{P}_{n}(x+1)\right)+D(x)\left(\check{P}_{n}(x)-\check{P}_{n}(x-1)\right)=\mathcal{E}_{n} \check{P}_{n}(x) \quad(\forall x \in \mathbb{C})
$$

\subsection{Original (q-)Racah system}

Let us consider the Racah $(\mathrm{R})$ and the $q$-Racah $(q \mathrm{R})$ cases. We follow the notation of [2]. Although there are four possible parameter choices indexed by $\left(\epsilon, \epsilon^{\prime}\right)=( \pm 1, \pm 1)$ in general, as explained in detail in $\S$ V.A.1 and $\S$ V.A.5 of [2], we restrict ourselves to the $\left(\epsilon, \epsilon^{\prime}\right)=(1,1)$ case for simplicity of presentation. The set of parameters $\boldsymbol{\lambda}$, which is different from the standard one $(\alpha, \beta, \gamma, \delta)[5]$, its shift $\boldsymbol{\delta}$ and $\kappa$ are

$$
\begin{aligned}
\mathrm{R}: \boldsymbol{\lambda}=(a, b, c, d), \quad \boldsymbol{\delta}=(1,1,1,1), \quad \kappa=1, \\
q \mathrm{R}: q^{\boldsymbol{\lambda}}=(a, b, c, d), \quad \boldsymbol{\delta}=(1,1,1,1), \quad \kappa=q^{-1}, \quad 0<q<1,
\end{aligned}
$$

where $q^{\boldsymbol{\lambda}}$ stands for $q^{\left(\lambda_{1}, \lambda_{2}, \ldots\right)}=\left(q^{\lambda_{1}}, q^{\lambda_{2}}, \ldots\right)$. We introduce a new parameter $\tilde{d}$ defined by

$$
\tilde{d} \stackrel{\text { def }}{=}\left\{\begin{array}{ll}
a+b+c-d-1 & : \mathrm{R} \\
a b c d^{-1} q^{-1} & : q \mathrm{R}
\end{array} .\right.
$$

We adopt the following choice of the parameter ranges:

$$
\mathrm{R}: \quad a=-N, \quad 0<d<a+b, \quad 0<c<1+d,
$$




$$
q \mathrm{R}: \quad a=q^{-N}, \quad 0<a b<d<1, \quad q d<c<1
$$

and $x_{\max }=n_{\max }=N$. They are sufficient for the positivity of $B(x ; \boldsymbol{\lambda})$ and $D(x ; \boldsymbol{\lambda})$ below.

Here are the fundamental data [2]:

$$
\begin{aligned}
& B(x ; \boldsymbol{\lambda})=\left\{\begin{array}{ll}
-\frac{(x+a)(x+b)(x+c)(x+d)}{(2 x+d)(2 x+1+d)} & : \mathrm{R} \\
-\frac{\left(1-a q^{x}\right)\left(1-b q^{x}\right)\left(1-c q^{x}\right)\left(1-d q^{x}\right)}{\left(1-d q^{2 x}\right)\left(1-d q^{2 x+1}\right)} & : q \mathrm{R}
\end{array},\right. \\
& D(x ; \boldsymbol{\lambda})=\left\{\begin{array}{ll}
-\frac{(x+d-a)(x+d-b)(x+d-c) x}{(2 x-1+d)(2 x+d)} & : \mathrm{R} \\
-\tilde{d} \frac{\left(1-a^{-1} d q^{x}\right)\left(1-b^{-1} d q^{x}\right)\left(1-c^{-1} d q^{x}\right)\left(1-q^{x}\right)}{\left(1-d q^{2 x-1}\right)\left(1-d q^{2 x}\right)} & : q \mathrm{R}
\end{array},\right. \\
& \mathcal{H}(\boldsymbol{\lambda}) \phi_{n}(x ; \boldsymbol{\lambda})=\mathcal{E}_{n}(\boldsymbol{\lambda}) \phi_{n}(x ; \boldsymbol{\lambda}) \quad\left(x=0,1, \ldots, x_{\max } ; n=0,1, \ldots, n_{\max }\right), \\
& \phi_{n}(x ; \boldsymbol{\lambda})=\phi_{0}(x ; \boldsymbol{\lambda}) \check{P}_{n}(x ; \boldsymbol{\lambda}), \\
& \mathcal{E}_{n}(\boldsymbol{\lambda})=\left\{\begin{array}{ll}
n(n+\tilde{d}) & : \mathrm{R} \\
\left(q^{-n}-1\right)\left(1-\tilde{d} q^{n}\right) & : q \mathrm{R}
\end{array}, \quad \eta(x ; \boldsymbol{\lambda})=\left\{\begin{array}{ll}
x(x+d) & : \mathrm{R} \\
\left(q^{-x}-1\right)\left(1-d q^{x}\right) & : q \mathrm{R}
\end{array},\right.\right. \\
& \varphi(x ; \boldsymbol{\lambda})=\left\{\begin{array}{ll}
\frac{2 x+d+1}{d+1} & : \mathrm{R} \\
\frac{q^{-x}-d q^{x+1}}{1-d q} & : q \mathrm{R}
\end{array},\right. \\
& \check{P}_{n}(x ; \boldsymbol{\lambda})=P_{n}(\eta(x ; \boldsymbol{\lambda}) ; \boldsymbol{\lambda})=\left\{\begin{array}{cc}
{ }_{4} F_{3}\left(\begin{array}{c}
-n, n+\tilde{d},-x, x+d \\
a, b, c
\end{array}\right) & : \mathrm{R} \\
{ }_{4} \phi_{3}\left(\begin{array}{c}
q^{-n}, \tilde{d} q^{n}, q^{-x}, d q^{x} \\
a, b, c
\end{array} \mid q ; q\right) & : q \mathrm{R}
\end{array}\right. \\
& =\left\{\begin{array}{ll}
R_{n}(\eta(x ; \boldsymbol{\lambda}) ; a-1, \tilde{d}-a, c-1, d-c) & : \mathrm{R} \\
R_{n}\left(1+d+\eta(x ; \boldsymbol{\lambda}) ; a q^{-1}, \tilde{d} a^{-1}, c q^{-1}, d c^{-1} \mid q\right) & : q \mathrm{R}
\end{array},\right. \\
& \phi_{0}(x ; \boldsymbol{\lambda})^{2}=\left\{\begin{array}{ll}
\frac{(a, b, c, d)_{x}}{(1+d-a, 1+d-b, 1+d-c, 1)_{x}} \frac{2 x+d}{d} & : \mathrm{R} \\
\frac{(a, b, c, d ; q)_{x}}{\left(a^{-1} d q, b^{-1} d q, c^{-1} d q, q ; q\right)_{x} \tilde{d}^{x}} \frac{1-d q^{2 x}}{1-d} & : q \mathrm{R}
\end{array},\right. \\
& d_{n}(\boldsymbol{\lambda})^{2}=\left\{\begin{array}{c}
\frac{(a, b, c, \tilde{d})_{n}}{(1+\tilde{d}-a, 1+\tilde{d}-b, 1+\tilde{d}-c, 1)_{n}} \frac{2 n+\tilde{d}}{\tilde{d}} \\
\times \frac{(-1)^{N}(1+d-a, 1+d-b, 1+d-c)_{N}}{(\tilde{d}+1)_{N}(d+1)_{2 N}} \\
\frac{(a, b, c, \tilde{d} ; q)_{n}}{\left(a^{-1} \tilde{d} q, b^{-1} \tilde{d} q, c^{-1} \tilde{d} q, q ; q\right)_{n} d^{n}} \frac{1-\tilde{d} q^{2 n}}{1-\tilde{d}} \\
\times \frac{(-1)^{N}\left(a^{-1} d q, b^{-1} d q, c^{-1} d q ; q\right)_{N} \tilde{d}^{N} q^{\frac{1}{2} N(N+1)}}{(\tilde{d} q ; q)_{N}(d q ; q)_{2 N}}
\end{array}\right.
\end{aligned}
$$

Here $R_{n}(\cdots)$ in (2.27) are the standard notation of the $(q-)$ Racah polynomial in [5]. It should 
be emphasised that the quantities $B(x ; \boldsymbol{\lambda}), D(x ; \boldsymbol{\lambda}), \mathcal{E}_{n}(\boldsymbol{\lambda}), \check{P}_{n}(x ; \boldsymbol{\lambda}), \phi_{0}(x ; \boldsymbol{\lambda})^{2}, d_{n}(\boldsymbol{\lambda})^{2}$ are formally symmetric under the permutation of $(a, b, c)$, although their ranges are restricted as above by (2.18) $-(2.19)$.

Here is a remark on the polynomial $\check{P}_{n}(x ; \boldsymbol{\lambda})$, which is in fact a polynomial in the sinusoidal coordinate $\eta(x ; \boldsymbol{\lambda})(2.24)$. The sinusoidal coordinate has a special dynamical meaning [2, 28, 43]. The Heisenberg operator solution for $\eta(x ; \boldsymbol{\lambda})$ can be expressed in a closed form. This means that its time evolution is a sinusoidal motion. Let $R$ be the ring of polynomials in $x$ (the Racah case) or the ring of Laurent polynomials in $q^{x}$ (the $q$-Racah case). Let us introduce an automorphism $\mathcal{I}$ in $R$ by

$$
\mathcal{I}(x)=-x-d \quad: \mathrm{R}, \quad \mathcal{I}\left(q^{x}\right)=q^{-x} d^{-1} \quad: q \mathrm{R} .
$$

Obviously it is an involution $\mathcal{I}^{2}=\mathrm{id}$. The following remark is important.

Remark: If a (Laurent) polynomial $\check{f}$ in $x\left(q^{x}\right)$ is invariant under the above involution, it is a polynomial in the sinusoidal coordinate $\eta(x ; \boldsymbol{\lambda})$ :

$$
\mathcal{I}(\check{f}(x))=\check{f}(x) \Leftrightarrow \check{f}(x)=f(\eta(x ; \boldsymbol{\lambda})) .
$$

The system is shape invariant [44, 2],

$$
\mathcal{A}(\boldsymbol{\lambda}) \mathcal{A}(\boldsymbol{\lambda})^{\dagger}=\kappa \mathcal{A}(\boldsymbol{\lambda}+\boldsymbol{\delta})^{\dagger} \mathcal{A}(\boldsymbol{\lambda}+\boldsymbol{\delta})+\mathcal{E}_{1}(\boldsymbol{\lambda})
$$

which is a sufficient condition for exact solvability and it provides the explicit formulas for the energy eigenvalues and the eigenfunctions, i.e. the generalised Rodrigues formula [2]. The forward and backward shift relations are

$$
\mathcal{F}(\boldsymbol{\lambda}) \check{P}_{n}(x ; \boldsymbol{\lambda})=\mathcal{E}_{n}(\boldsymbol{\lambda}) \check{P}_{n-1}(x ; \boldsymbol{\lambda}+\boldsymbol{\delta}), \quad \mathcal{B}(\boldsymbol{\lambda}) \check{P}_{n-1}(x ; \boldsymbol{\lambda}+\boldsymbol{\delta})=\check{P}_{n}(x ; \boldsymbol{\lambda}),
$$

where the forward and backward shift operators are

$$
\mathcal{F}(\boldsymbol{\lambda})=B(0 ; \boldsymbol{\lambda}) \varphi(x ; \boldsymbol{\lambda})^{-1}\left(1-e^{\partial}\right), \quad \mathcal{B}(\boldsymbol{\lambda})=B(0 ; \boldsymbol{\lambda})^{-1}\left(B(x ; \boldsymbol{\lambda})-D(x ; \boldsymbol{\lambda}) e^{-\partial}\right) \varphi(x ; \boldsymbol{\lambda})
$$

\subsection{Symmetry and virtual state vectors}

Let us define the twist operation $\mathfrak{t}$ of the parameters:

$$
\mathfrak{t}(\boldsymbol{\lambda}) \stackrel{\text { def }}{=}\left(\lambda_{4}-\lambda_{1}+1, \lambda_{4}-\lambda_{2}+1, \lambda_{3}, \lambda_{4}\right), \quad \mathfrak{t}^{2}=\mathrm{id}
$$


We introduce two functions $B^{\prime}(x)$ and $D^{\prime}(x)$ by

$$
B^{\prime}(x ; \boldsymbol{\lambda}) \stackrel{\text { def }}{=} B(x ; \mathfrak{t}(\boldsymbol{\lambda})), \quad D^{\prime}(x ; \boldsymbol{\lambda}) \stackrel{\text { def }}{=} D(x ; \mathfrak{t}(\boldsymbol{\lambda})),
$$

namely,

$$
\begin{aligned}
& B^{\prime}(x ; \boldsymbol{\lambda})=\left\{\begin{array}{ll}
-\frac{(x+d-a+1)(x+d-b+1)(x+c)(x+d)}{(2 x+d)(2 x+1+d)} & : \mathrm{R} \\
-\frac{\left(1-a^{-1} d q^{x+1}\right)\left(1-b^{-1} d q^{x+1}\right)\left(1-c q^{x}\right)\left(1-d q^{x}\right)}{\left(1-d q^{2 x}\right)\left(1-d q^{2 x+1}\right)} & : q \mathrm{R}
\end{array},\right. \\
& D^{\prime}(x ; \boldsymbol{\lambda})= \begin{cases}-\frac{(x+a-1)(x+b-1)(x+d-c) x}{(2 x-1+d)(2 x+d)} & : \mathrm{R} \\
-\frac{c d q}{a b} \frac{\left(1-a q^{x-1}\right)\left(1-b q^{x-1}\right)\left(1-c^{-1} d q^{x}\right)\left(1-q^{x}\right)}{\left(1-d q^{2 x-1}\right)\left(1-d q^{2 x}\right)} & : q \mathrm{R}\end{cases}
\end{aligned}
$$

We restrict the parameter range

$$
\mathrm{R}: \quad d+M<a+b, \quad q \mathrm{R}: \quad a b<d q^{M},
$$

in which $M$ is a positive integer and later it will be identified with the total number of deleted virtual states. It is easy to verify

$$
\begin{aligned}
& B(x ; \boldsymbol{\lambda}) D(x+1 ; \boldsymbol{\lambda})=\alpha(\boldsymbol{\lambda})^{2} B^{\prime}(x ; \boldsymbol{\lambda}) D^{\prime}(x+1 ; \boldsymbol{\lambda}), \\
& B(x ; \boldsymbol{\lambda})+D(x ; \boldsymbol{\lambda})=\alpha(\boldsymbol{\lambda})\left(B^{\prime}(x ; \boldsymbol{\lambda})+D^{\prime}(x ; \boldsymbol{\lambda})\right)+\alpha^{\prime}(\boldsymbol{\lambda}), \\
& B^{\prime}(x ; \boldsymbol{\lambda})>0 \quad\left(x=0,1, \ldots, x_{\max }+M-1\right), \\
& D^{\prime}(x ; \boldsymbol{\lambda})>0 \quad\left(x=1,2, \ldots, x_{\max }\right), \quad D^{\prime}(0 ; \boldsymbol{\lambda})=D^{\prime}\left(x_{\max }+1 ; \boldsymbol{\lambda}\right)=0 .
\end{aligned}
$$

Here the constant $\alpha(\boldsymbol{\lambda})$ is positive and $\alpha^{\prime}(\boldsymbol{\lambda})$ is negative:

$$
0<\alpha(\boldsymbol{\lambda})=\left\{\begin{array}{ll}
1 & : \mathrm{R} \\
a b d^{-1} q^{-1} & : q \mathrm{R}
\end{array} \quad, \quad 0>\alpha^{\prime}(\boldsymbol{\lambda})=\left\{\begin{array}{ll}
-c(a+b-d-1) & : \mathrm{R} \\
-(1-c)\left(1-a b d^{-1} q^{-1}\right) & : q \mathrm{R}
\end{array} .\right.\right.
$$

The above relations (2.40)-(2.43) imply that we can define a virtual Hamiltonian $\mathcal{H}^{\prime}$ by the twisted parameters (the $\boldsymbol{\lambda}$ dependence is suppressed for simplicity):

$$
\mathcal{H}^{\prime}(\boldsymbol{\lambda}) \stackrel{\text { def }}{=} \mathcal{H}(\mathfrak{t}(\boldsymbol{\lambda}))=-\sqrt{B^{\prime}(x)} e^{\partial} \sqrt{D^{\prime}(x)}-\sqrt{D^{\prime}(x)} e^{-\partial} \sqrt{B^{\prime}(x)}+B^{\prime}(x)+D^{\prime}(x)
$$

and the original Hamiltonian and the virtual Hamiltonian are linearly related

$$
\mathcal{H}(\boldsymbol{\lambda})=\alpha(\boldsymbol{\lambda}) \mathcal{H}(\mathfrak{t}(\boldsymbol{\lambda}))+\alpha^{\prime}(\boldsymbol{\lambda}) .
$$


This also means that $\mathcal{H}(\mathfrak{t}(\boldsymbol{\lambda}))$ is positive definite and it has no zero-mode. In other words, the two term recurrence relation determining the 'zero-mode' of $\mathcal{H}(\mathfrak{t}(\boldsymbol{\lambda})$ )

$$
\begin{aligned}
& \mathcal{A}(\mathfrak{t}(\boldsymbol{\lambda}))=\sqrt{B^{\prime}(x ; \boldsymbol{\lambda})}-e^{\partial} \sqrt{D^{\prime}(x ; \boldsymbol{\lambda})}, \\
& \mathcal{A}(\mathfrak{t}(\boldsymbol{\lambda})) \tilde{\phi}_{0}(x ; \boldsymbol{\lambda})=0 \quad\left(x=0,1, \ldots, x_{\max }-1\right),
\end{aligned}
$$

can be 'solved' from $x=0$ to $x=x_{\max }-1$ to determine

$$
\tilde{\phi}_{0}(x ; \boldsymbol{\lambda}) \stackrel{\text { def }}{=} \sqrt{\prod_{y=0}^{x-1} \frac{B^{\prime}(y ; \boldsymbol{\lambda})}{D^{\prime}(y+1 ; \boldsymbol{\lambda})}} \quad\left(x=0,1, \ldots, x_{\max }\right) .
$$

But at the end point $x=x_{\max }$, the 'zero-mode' equation (2.47) is not satisfied, because of the boundary condition

$$
B^{\prime}\left(x_{\max } ; \boldsymbol{\lambda}\right) \neq 0, \quad \mathcal{A}(\mathfrak{t}(\boldsymbol{\lambda})) \tilde{\phi}_{0}(x ; \boldsymbol{\lambda}) \neq 0 \quad\left(x=x_{\max }\right) .
$$

The new Schrödinger equation

$$
\mathcal{H}^{\prime}(\boldsymbol{\lambda}) \tilde{\phi}_{\mathrm{v}}(x ; \boldsymbol{\lambda})=\mathcal{E}_{\mathrm{v}}^{\prime}(\boldsymbol{\lambda}) \tilde{\phi}_{\mathrm{v}}(x ; \boldsymbol{\lambda})
$$

can be almost solved except for the end point $x=x_{\max }$ by the factorisation ansatz

$$
\tilde{\phi}_{\mathrm{v}}(x ; \boldsymbol{\lambda}) \stackrel{\text { def }}{=} \tilde{\phi}_{0}(x ; \boldsymbol{\lambda}) \check{\xi}_{\mathrm{v}}(x ; \boldsymbol{\lambda})
$$

as in the original $(q-)$ Racah system. By using the explicit form of $\tilde{\phi}_{0}(x ; \boldsymbol{\lambda})(2.48)$, the new Schrödinger equation for $x=0, \ldots, x_{\max }-1$ is rewritten as

$$
B^{\prime}(x ; \boldsymbol{\lambda})\left(\check{\xi}_{\mathrm{v}}(x ; \boldsymbol{\lambda})-\check{\xi}_{\mathrm{v}}(x+1 ; \boldsymbol{\lambda})\right)+D^{\prime}(x ; \boldsymbol{\lambda})\left(\check{\xi}_{\mathrm{v}}(x ; \boldsymbol{\lambda})-\check{\xi}_{\mathrm{v}}(x-1 ; \boldsymbol{\lambda})\right)=\mathcal{E}_{\mathrm{v}}^{\prime}(\boldsymbol{\lambda}) \check{\xi}_{\mathrm{v}}(x ; \boldsymbol{\lambda})
$$

This is the same form of equation as that for the $(q-)$ Racah polynomials. So its solution for $x \in \mathbb{C}$ is given by the $(q-)$ Racah polynomial (2.26) with the twisted parameters:

$$
\check{\xi}_{\mathrm{v}}(x ; \boldsymbol{\lambda})=\check{P}_{\mathrm{v}}(x ; \mathfrak{t}(\boldsymbol{\lambda})), \quad \mathcal{E}_{\mathrm{v}}^{\prime}(\boldsymbol{\lambda})=\mathcal{E}_{\mathrm{v}}(\mathfrak{t}(\boldsymbol{\lambda}))
$$

Among such 'solutions', those with the negative energy and having definite sign

$$
\begin{aligned}
\check{\xi}_{\mathrm{v}}(x ; \boldsymbol{\lambda})>0 \quad\left(x=0,1, \ldots, x_{\max }, x_{\max }+1 ; \mathrm{v} \in \mathcal{V}\right), \\
\tilde{\mathcal{E}}_{\mathrm{v}}(\boldsymbol{\lambda})<0 \quad(\mathrm{v} \in \mathcal{V}),
\end{aligned}
$$


are called the virtual state vectors: $\left\{\tilde{\phi}_{\mathrm{v}}(x)\right\}, \mathrm{v} \in \mathcal{V}$. The index set of the virtual state vectors is

$$
\mathcal{V}=\left\{1,2, \ldots, \mathrm{v}_{\max }\right\}, \quad \mathrm{v}_{\max }=\min \left\{\left[\lambda_{1}+\lambda_{2}-\lambda_{4}-1\right]^{\prime},\left[\frac{1}{2}\left(\lambda_{1}+\lambda_{2}-\lambda_{3}-\lambda_{4}\right)\right]\right\}
$$

where $[x]$ denotes the greatest integer not exceeding $x$ and $[x]^{\prime}$ denotes the greatest integer not equal or exceeding $x$. We will not use the label 0 state for deletion, see (3.58). The negative virtual state energy conditions (2.54) is met by $\mathrm{v}_{\max } \leq\left[\lambda_{1}+\lambda_{2}-\lambda_{4}-1\right]^{\prime}$. For the positivity of $\check{\xi}_{\mathrm{v}}(x ; \boldsymbol{\lambda})(2.53)$, we write down them explicitly:

$$
\begin{aligned}
& \check{\xi}_{\mathrm{v}}(x ; \boldsymbol{\lambda})=\left\{\begin{array}{cc}
{ }_{4} F_{3}\left(\begin{array}{c}
-\mathrm{v}, \mathrm{v}-a-b+c+d+1,-x, x+d \\
d-a+1, d-b+1, c
\end{array} \mid 1\right) & : \mathrm{R} \\
{ }_{4} \phi_{3}\left(\begin{array}{c}
q^{-\mathrm{v}}, a^{-1} b^{-1} c d q^{\mathrm{v}+1}, q^{-x}, d q^{x} \mid \\
a^{-1} d q, b^{-1} d q, c
\end{array} \mid q\right) & : q \mathrm{R}
\end{array}\right. \\
&= \begin{cases}\sum_{k=0}^{\mathrm{v}} \frac{(-\mathrm{v}, \mathrm{v}-a-b+c+d+1,-x, x+d)_{k}}{(d-a+1, d-b+1, c)_{k}} \frac{1}{k !} & : \mathrm{R} \\
\sum_{k=0}^{\mathrm{v}} \frac{\left(q^{-\mathrm{v}}, a^{-1} b^{-1} c d q^{\mathrm{v}+1}, q^{-x}, d q^{x} ; q\right)_{k}}{\left(a^{-1} d q, b^{-1} d q, c ; q\right)_{k}} \frac{q^{k}}{(q ; q)_{k}} & : q \mathrm{R}\end{cases}
\end{aligned}
$$

Each $k$-th term in the sum is non-negative for $2 \mathrm{v}_{\max } \leq \lambda_{1}+\lambda_{2}-\lambda_{3}-\lambda_{4}$.

Here is a summary of the properties of the virtual state vectors:

$$
\begin{aligned}
& \tilde{\phi}_{0}(x ; \boldsymbol{\lambda}) \stackrel{\text { def }}{=} \phi_{0}(x ; \mathfrak{t}(\boldsymbol{\lambda})), \quad \tilde{\phi}_{\mathrm{v}}(x ; \boldsymbol{\lambda}) \stackrel{\text { def }}{=} \phi_{\mathrm{v}}(x ; \mathfrak{t}(\boldsymbol{\lambda}))=\tilde{\phi}_{0}(x ; \boldsymbol{\lambda}) \check{\xi}_{\mathrm{v}}(x ; \boldsymbol{\lambda}) \quad(\mathrm{v} \in \mathcal{V}), \\
& \check{\xi}_{\mathrm{v}}(x ; \boldsymbol{\lambda}) \stackrel{\text { def }}{=} \check{P}_{\mathrm{v}}(x ; \mathfrak{t}(\boldsymbol{\lambda})), \quad \check{\xi}_{\mathrm{v}}(x ; \boldsymbol{\lambda}) \stackrel{\text { def }}{=} \xi_{\mathrm{v}}(\eta(x ; \boldsymbol{\lambda}) ; \boldsymbol{\lambda}), \\
& \mathcal{H}(\boldsymbol{\lambda}) \tilde{\phi}_{\mathrm{v}}(x ; \boldsymbol{\lambda})=\tilde{\mathcal{E}}_{\mathrm{v}}(\boldsymbol{\lambda}) \tilde{\phi}_{\mathrm{v}}(x ; \boldsymbol{\lambda}) \quad\left(x=0,1, \ldots, x_{\max }-1\right), \\
& \mathcal{H}(\boldsymbol{\lambda}) \tilde{\phi}_{\mathrm{v}}\left(x_{\max } ; \boldsymbol{\lambda}\right) \neq \tilde{\mathcal{E}}_{\mathrm{v}}(\boldsymbol{\lambda}) \tilde{\phi}_{\mathrm{v}}\left(x_{\max } ; \boldsymbol{\lambda}\right), \quad \mathcal{E}_{\mathrm{v}}^{\prime}(\boldsymbol{\lambda})=\mathcal{E}_{\mathrm{v}}(\mathfrak{t}(\boldsymbol{\lambda})), \\
& \tilde{\mathcal{E}}_{\mathrm{v}}(\boldsymbol{\lambda})=\alpha(\boldsymbol{\lambda}) \mathcal{E}_{\mathrm{v}}^{\prime}(\boldsymbol{\lambda})+\alpha^{\prime}(\boldsymbol{\lambda})=\left\{\begin{array}{ll}
-(c+\mathrm{v})(a+b-d-1-\mathrm{v}) \quad: \mathrm{R} \\
-\left(1-c q^{\mathrm{v}}\right)\left(1-a b d^{-1} q^{-1-\mathrm{v}}\right) \quad: q \mathrm{R}
\end{array},\right. \\
& \nu(x ; \boldsymbol{\lambda}) \stackrel{\text { def }}{=} \frac{\phi_{0}(x ; \boldsymbol{\lambda})}{\tilde{\phi}_{0}(x ; \boldsymbol{\lambda})}=\left\{\begin{array}{l}
\frac{\Gamma(1-a) \Gamma(x+b) \Gamma(d-a+1) \Gamma(b-d-x)}{\Gamma(1-a-x) \Gamma(b) \Gamma(x+d-a+1) \Gamma(b-d)}: \mathrm{R} \\
\frac{\left(a^{-1} q^{1-x}, b, a^{-1} d q^{x+1}, b d^{-1} ; q\right)_{\infty}}{\left(a^{-1} q, b q^{x}, a^{-1} d q, b d^{-1} q^{-x} ; q\right)_{\infty}}
\end{array}\right.
\end{aligned}
$$

Note that $\alpha^{\prime}(\boldsymbol{\lambda})=\tilde{\mathcal{E}}_{0}(\boldsymbol{\lambda})<0$. The function $\nu(x ; \boldsymbol{\lambda})$ can be analytically continued into a meromorphic function of $x$ or $q^{x}$ through the functional relations:

$$
\nu(x+1 ; \boldsymbol{\lambda})=\frac{B(x ; \boldsymbol{\lambda})}{\alpha B^{\prime}(x ; \boldsymbol{\lambda})} \nu(x ; \boldsymbol{\lambda}), \quad \nu(x-1 ; \boldsymbol{\lambda})=\frac{D(x ; \boldsymbol{\lambda})}{\alpha D^{\prime}(x ; \boldsymbol{\lambda})} \nu(x ; \boldsymbol{\lambda}) .
$$

By $B\left(x_{\max } ; \boldsymbol{\lambda}\right)=0$, it vanishes for integer $x_{\max }+1 \leq x \leq x_{\max }+M, \nu(x ; \boldsymbol{\lambda})=0$, and at negative integer points it takes nonzero finite values in general. 


\section{Multi-indexed ( $q$-) Racah Polynomials}

In this section we apply the Crum-Adler method of virtual states deletion to the exactly solvable systems whose eigenstates are described by the $(q-)$ Racah polynomials. Since all the eigenvalues remain the same, i.e. the process is exactly iso-spectral deformation, the size of the Hamiltonian is unchanged.

Various quantities are neatly expressed in terms of a Casoratian, a discrete counterpart of the Wronskian. The Casorati determinant of a set of $n$ functions $\left\{f_{j}(x)\right\}$ is defined by

$$
\mathrm{W}\left[f_{1}, \ldots, f_{n}\right](x) \stackrel{\text { def }}{=} \operatorname{det}\left(f_{k}(x+j-1)\right)_{1 \leq j, k \leq n},
$$

(for $n=0$, we set $\mathrm{W}[\cdot](x)=1$ ), which satisfies identities

$$
\begin{aligned}
& \mathrm{W}\left[g f_{1}, g f_{2}, \ldots, g f_{n}\right](x)=\prod_{k=0}^{n-1} g(x+k) \cdot \mathrm{W}\left[f_{1}, f_{2}, \ldots, f_{n}\right](x), \\
& \mathrm{W}\left[\mathrm{W}\left[f_{1}, f_{2}, \ldots, f_{n}, g\right], \mathrm{W}\left[f_{1}, f_{2}, \ldots, f_{n}, h\right]\right](x) \\
& =\mathrm{W}\left[f_{1}, f_{2}, \ldots, f_{n}\right](x+1) \mathrm{W}\left[f_{1}, f_{2}, \ldots, f_{n}, g, h\right](x) \quad(n \geq 0) .
\end{aligned}
$$

\subsection{Virtual states deletion}

Let us provide the basic formulas starting from one virtual state deletion. For simplicity of presentation the parameter $(\boldsymbol{\lambda})$ dependence of various quantities is suppressed in this subsection.

one virtual state vector deletion

First we rewrite the original Hamiltonian by introducing potential functions $\hat{B}_{d_{1}}(x)$ and $\hat{D}_{d_{1}}(x)$ determined by one of the virtual state polynomials $\check{\xi}_{d_{1}}(x)\left(d_{1} \in \mathcal{V}\right)$ :

$$
\hat{B}_{d_{1}}(x) \stackrel{\text { def }}{=} \alpha B^{\prime}(x) \frac{\check{\xi}_{d_{1}}(x+1)}{\check{\xi}_{d_{1}}(x)}, \quad \hat{D}_{d_{1}}(x) \stackrel{\text { def }}{=} \alpha D^{\prime}(x) \frac{\check{\xi}_{d_{1}}(x-1)}{\check{\xi}_{d_{1}}(x)} .
$$

We have $\hat{B}_{d_{1}}(x)>0\left(x=0,1, \ldots, x_{\max }\right), \hat{D}_{d_{1}}(0)=\hat{D}_{d_{1}}\left(x_{\max }+1\right)=0, \hat{D}_{d_{1}}(x)>0(x=$ $\left.1,2, \ldots, x_{\max }\right)$ and

$$
\begin{aligned}
& B(x) D(x+1)=\hat{B}_{d_{1}}(x) \hat{D}_{d_{1}}(x+1), \\
& B(x)+D(x)=\hat{B}_{d_{1}}(x)+\hat{D}_{d_{1}}(x)+\tilde{\mathcal{E}}_{d_{1}},
\end{aligned}
$$

where use is made of (2.51) in the second equation. The original Hamiltonian reads:

$$
\mathcal{H}=\hat{\mathcal{A}}_{d_{1}}^{\dagger} \hat{\mathcal{A}}_{d_{1}}+\tilde{\mathcal{E}}_{d_{1}}
$$




$$
\hat{\mathcal{A}}_{d_{1}} \stackrel{\text { def }}{=} \sqrt{\hat{B}_{d_{1}}(x)}-e^{\partial} \sqrt{\hat{D}_{d_{1}}(x)}, \quad \hat{\mathcal{A}}_{d_{1}}^{\dagger}=\sqrt{\hat{B}_{d_{1}}(x)}-\sqrt{\hat{D}_{d_{1}}(x)} e^{-\partial}
$$

The virtual state vector $\tilde{\phi}_{d_{1}}(x)$ is almost annihilated by $\hat{\mathcal{A}}_{d_{1}}$, except for the upper end point:

$$
\hat{\mathcal{A}}_{d_{1}} \tilde{\phi}_{d_{1}}(x)=0 \quad\left(x=0,1, \ldots, x_{\max }-1\right), \quad \hat{\mathcal{A}}_{d_{1}} \tilde{\phi}_{d_{1}}\left(x_{\max }\right) \neq 0 .
$$

The proof is straightforward by direct substitution of (3.1) and (2.48).

Next let us define a new Hamiltonian $\mathcal{H}_{d_{1}}$ by changing the order of the two matrices $\hat{\mathcal{A}}_{d_{1}}^{\dagger}$ and $\hat{\mathcal{A}}_{d_{1}}$ together with the sets of new eigenvectors $\phi_{d_{1} n}(x)$ and new virtual state vectors $\tilde{\phi}_{d_{1 \mathrm{v}}}(x)$ :

$$
\begin{aligned}
\mathcal{H}_{d_{1}} & \stackrel{\text { def }}{=} \hat{\mathcal{A}}_{d_{1}} \hat{\mathcal{A}}_{d_{1}}^{\dagger}+\tilde{\mathcal{E}}_{d_{1}}, \quad \mathcal{H}_{d_{1}}=\left(\mathcal{H}_{d_{1} x, y}\right) \quad\left(x, y=0,1, \ldots, x_{\max }\right), \\
\phi_{d_{1} n}(x) & \stackrel{\text { def }}{=} \hat{\mathcal{A}}_{d_{1}} \phi_{n}(x) \quad\left(x=0,1, \ldots, x_{\max } ; n=0,1, \ldots, n_{\max }\right), \\
\tilde{\phi}_{d_{1} \mathrm{v}}(x) & \stackrel{\text { def }}{=} \hat{\mathcal{A}}_{d_{1}} \tilde{\phi}_{\mathrm{v}}(x)+\delta_{x, x_{\max }} \varphi_{d_{1} \mathrm{v}}\left(x=0,1, \ldots, x_{\max } ; \mathrm{v} \in \mathcal{V} \backslash\left\{d_{1}\right\}\right), \\
\varphi_{d_{1} \mathrm{v}} & \stackrel{\text { def }}{=}-\frac{\sqrt{\alpha B^{\prime}\left(x_{\max }\right)} \tilde{\phi}_{0}\left(x_{\max }\right)}{\sqrt{\check{\xi}_{d_{1}}\left(x_{\max }\right) \check{\xi}_{d_{1}}\left(x_{\max }+1\right)}} \check{\xi}_{d_{1}}\left(x_{\max }\right) \check{\xi}_{\mathrm{v}}\left(x_{\max }+1\right) .
\end{aligned}
$$

The $\varphi_{d_{1 \mathrm{v}}}$ term is necessary for the Casoratian expression for $\tilde{\phi}_{d_{1} \mathrm{v}}(x)$ in (3.5) to hold at $x=x_{\max }$. It is easy to verify that $\phi_{d_{1} n}(x)$ is an eigenvector and that $\tilde{\phi}_{d_{1 \mathrm{v}}}(x)$ is a virtual state vector

$$
\begin{aligned}
& \mathcal{H}_{d_{1}} \phi_{d_{1} n}(x)=\mathcal{E}_{n} \phi_{d_{1} n}(x) \quad\left(x=0,1, \ldots, x_{\max } ; n=0,1, \ldots, n_{\max }\right), \\
& \mathcal{H}_{d_{1}} \tilde{\phi}_{d_{1 \mathrm{v}}}(x)=\tilde{\mathcal{E}}_{\mathrm{v}} \tilde{\phi}_{d_{1 \mathrm{v}}}(x) \quad\left(x=0,1, \ldots, x_{\max }-1 ; \mathrm{v} \in \mathcal{V} \backslash\left\{d_{1}\right\}\right), \\
& \mathcal{H}_{d_{1}} \tilde{\phi}_{d_{1 \mathrm{v}}}\left(x_{\max }\right) \neq \tilde{\mathcal{E}}_{\mathrm{v}} \tilde{\phi}_{d_{1} \mathrm{v}}\left(x_{\max }\right) .
\end{aligned}
$$

For example,

$$
\begin{aligned}
& \mathcal{H}_{d_{1}} \phi_{d_{1} n}=\left(\hat{\mathcal{A}}_{d_{1}} \hat{\mathcal{A}}_{d_{1}}^{\dagger}+\tilde{\mathcal{E}}_{d_{1}}\right) \hat{\mathcal{A}}_{d_{1}} \phi_{n}=\hat{\mathcal{A}}_{d_{1}}\left(\hat{\mathcal{A}}_{d_{1}}^{\dagger} \hat{\mathcal{A}}_{d_{1}}+\tilde{\mathcal{E}}_{d_{1}}\right) \phi_{n} \\
& =\hat{\mathcal{A}}_{d_{1}} \mathcal{H} \phi_{n}=\hat{\mathcal{A}}_{d_{1}} \mathcal{E}_{n} \phi_{n}=\mathcal{E}_{n} \hat{\mathcal{A}}_{d_{1}} \phi_{n}=\mathcal{E}_{n} \phi_{d_{1} n} .
\end{aligned}
$$

The two Hamiltonians $\mathcal{H}$ and $\mathcal{H}_{d_{1}}$ are exactly iso-spectral. If the original system is exactly solvable, this new system is also exactly solvable. The orthogonality relation for the new eigenvectors is

$$
\left(\phi_{d_{1} n}, \phi_{d_{1} m}\right)=\sum_{x=0}^{x_{\max }} \phi_{d_{1} n}(x) \phi_{d_{1} m}(x)
$$




$$
\begin{aligned}
& =\left(\hat{\mathcal{A}}_{d_{1}} \phi_{n}, \hat{\mathcal{A}}_{d_{1}} \phi_{m}\right)=\left(\hat{\mathcal{A}}_{d_{1}}^{\dagger} \hat{\mathcal{A}}_{d_{1}} \phi_{n}, \phi_{m}\right)=\left(\left(\mathcal{H}-\tilde{\mathcal{E}}_{d_{1}}\right) \phi_{n}, \phi_{m}\right) \\
& =\left(\mathcal{E}_{n}-\tilde{\mathcal{E}}_{d_{1}}\right)\left(\phi_{n}, \phi_{m}\right)=\left(\mathcal{E}_{n}-\tilde{\mathcal{E}}_{d_{1}}\right) \frac{1}{d_{n}^{2}} \delta_{n m} \quad\left(n, m=0,1, \ldots, n_{\max }\right) .
\end{aligned}
$$

This shows clearly that the negative virtual state energy $\left(\tilde{\mathcal{E}}_{\mathrm{v}}<0\right)$ is necessary for the positivity of the inner products.

The new eigenvector $\phi_{d_{1} n}(x)(3.3)$ and the virtual state vector $\tilde{\phi}_{d_{1 \mathrm{v}}}(x)$ (3.4) are expressed neatly in terms of the Casoratian $\left(x=0,1, \ldots, x_{\max }\right)$

$$
\phi_{d_{1} n}(x)=\frac{-\sqrt{\alpha B^{\prime}(x)} \tilde{\phi}_{0}(x)}{\sqrt{\check{\xi}_{d_{1}}(x) \check{\xi}_{d_{1}}(x+1)}} \mathrm{W}\left[\check{\xi}_{d_{1}}, \nu \check{P}_{n}\right](x), \quad \tilde{\phi}_{d_{1} \mathrm{v}}(x)=\frac{-\sqrt{\alpha B^{\prime}(x)} \tilde{\phi}_{0}(x)}{\sqrt{\check{\xi}_{d_{1}}(x) \check{\xi}_{d_{1}}(x+1)}} \mathrm{W}\left[\check{\xi}_{d_{1}}, \check{\xi}_{\mathrm{v}}\right](x) .
$$

We will show that the positivity of the virtual state vector is inherited by the new virtual state vector $\tilde{\phi}_{d_{1 \mathrm{v}}}(x)(\underline{3.4})$. The Casoratian $\mathrm{W}\left[\check{\xi}_{d_{1}}, \check{\xi}_{\mathrm{v}}\right](x)$ has definite sign for $x=0,1, \ldots, x_{\max }+1$, namely all positive or all negative. By using (2.51) we have

$$
\alpha B^{\prime}(x) \mathrm{W}\left[\check{\xi}_{d_{1}}, \check{\xi}_{\mathrm{v}}\right](x)=\alpha D^{\prime}(x) \mathrm{W}\left[\check{\xi}_{d_{1}}, \check{\xi}_{\mathrm{v}}\right](x-1)+\left(\tilde{\mathcal{E}}_{d_{1}}-\tilde{\mathcal{E}}_{\mathrm{v}}\right) \check{\xi}_{d_{1}}(x) \check{\xi}_{\mathrm{v}}(x)
$$

By setting $x=0,1, \ldots, x_{\max }+1$ in turn, we obtain

$$
\pm\left(\tilde{\mathcal{E}}_{d_{1}}-\tilde{\mathcal{E}}_{\mathrm{v}}\right)>0 \Rightarrow \pm \mathrm{W}\left[\check{\xi}_{d_{1}}, \check{\xi}_{\mathrm{v}}\right](x)>0 \quad\left(x=0,1, \ldots, x_{\max }+1\right)
$$

Note that the set of virtual eigenvalues $\left\{\tilde{\mathcal{E}}_{d_{j}}\right\}$ are mutually distinct. We will now show that the new groundstate eigenvector $\phi_{d_{1} 0}(x)$ is of definite sign as the original one $\phi_{0}(x)$ (2.8). We show that the Casoratian $\mathrm{W}\left[\check{\xi}_{d_{1}}, \nu\right](x)$ has definite sign for $x=0,1, \ldots, x_{\max }$. By writing down the equation $\mathcal{H} \phi_{n}(x)=\mathcal{E}_{n} \phi_{n}(x)\left(x=0,1, \ldots, x_{\max }\right)$ with $\mathcal{H}=\hat{A}_{d_{1}}^{\dagger} \hat{A}_{d_{1}}+\tilde{\mathcal{E}}_{d_{1}}$ and (2.14), we have

$$
\alpha B^{\prime}(x) \nu(x+1) \check{P}_{n}(x+1)+\alpha D^{\prime}(x) \nu(x-1) \check{P}_{n}(x-1)=\left(B(x)+D(x)-\mathcal{E}_{n}\right) \nu(x) \check{P}_{n}(x) .
$$

In terms of the functional relations of $\nu(x)(2.62)$, it is reduced to the original difference equation for $\check{P}_{n}(x)$ and it is valid any $x \in \mathbb{C}$. By using this, we can show

$$
\alpha B^{\prime}(x) \mathrm{W}\left[\check{\xi}_{d_{1}}, \nu \check{P}_{n}\right](x)=\alpha D^{\prime}(x) \mathrm{W}\left[\check{\xi}_{d_{1}}, \nu \check{P}_{n}\right](x-1)+\left(\tilde{\mathcal{E}}_{d_{1}}-\mathcal{E}_{n}\right) \check{\xi}_{d_{1}}(x) \nu(x) \check{P}_{n}(x)
$$

By setting $n=0$ and $x=0,1, \ldots, x_{\max }$ in turn, we obtain

$$
-\mathrm{W}\left[\check{\xi}_{d_{1}}, \nu\right](x)>0 \quad\left(x=0,1, \ldots, x_{\max }\right) .
$$


Let us rewrite the deformed Hamiltonian $\mathcal{H}_{d_{1}}$ in the standard form. The potential functions $B_{d_{1}}(x)$ and $D_{d_{1}}(x)$ are introduced:

$$
\begin{aligned}
& B_{d_{1}}(x) \stackrel{\text { def }}{=} \alpha B^{\prime}(x+1) \frac{\check{\xi}_{d_{1}}(x)}{\check{\xi}_{d_{1}}(x+1)} \frac{\mathrm{W}\left[\check{\xi}_{d_{1}}, \nu\right](x+1)}{\mathrm{W}\left[\check{\xi}_{d_{1}}, \nu\right](x)}, \\
& D_{d_{1}}(x) \stackrel{\text { def }}{=} \alpha D^{\prime}(x) \frac{\check{\xi}_{d_{1}}(x+1)}{\check{\xi}_{d_{1}}(x)} \frac{\mathrm{W}\left[\check{\xi}_{d_{1}}, \nu\right](x-1)}{\mathrm{W}\left[\check{\xi}_{d_{1}}, \nu\right](x)} .
\end{aligned}
$$

The positivity of $B_{d_{1}}(x)$ and $D_{d_{1}}(x)$ is shown above and the boundary conditions $B_{d_{1}}\left(x_{\max }\right)=$ 0 and $D_{d_{1}}(0)=0$ are satisfied. They satisfy the relations

$$
\begin{aligned}
& B_{d_{1}}(x) D_{d_{1}}(x+1)=\hat{B}_{d_{1}}(x+1) \hat{D}_{d_{1}}(x+1), \\
& B_{d_{1}}(x)+D_{d_{1}}(x)=\hat{B}_{d_{1}}(x)+\hat{D}_{d_{1}}(x+1)+\tilde{\mathcal{E}}_{d_{1}} .
\end{aligned}
$$

The standard form Hamiltonian is obtained:

$$
\begin{aligned}
& \mathcal{H}_{d_{1}}=\mathcal{A}_{d_{1}}^{\dagger} \mathcal{A}_{d_{1}}, \\
& \mathcal{A}_{d_{1}} \stackrel{\text { def }}{=} \sqrt{B_{d_{1}}(x)}-e^{\partial} \sqrt{D_{d_{1}}(x)}, \quad \mathcal{A}_{d_{1}}^{\dagger}=\sqrt{B_{d_{1}}(x)}-\sqrt{D_{d_{1}}(x)} e^{-\partial},
\end{aligned}
$$

in which $\mathcal{A}_{d_{1}}$ annihilates the groundstate eigenvector

$$
\mathcal{A}_{d_{1}} \phi_{d_{1} 0}(x)=0 \quad\left(x=0,1, \ldots, x_{\max }\right) .
$$

This one virtual state vector deletion is essentially the same procedure as that developed for the exceptional orthogonal polynomials in [23]. See $\S 3.2$ for the explicit expressions. $\underline{\text { multi virtual state vector deletion }}$

We repeat the above procedure and obtain the modified systems. The number of deleted virtual state vectors should be less than or equal $|\mathcal{V}|$ and $M$.

Let us assume that we have already deleted $s$ virtual state vectors $(s \geq 1)$, which are labeled by $\left\{d_{1}, \ldots, d_{s}\right\}\left(d_{j} \in \mathcal{V}:\right.$ mutually distinct). Namely we have

$$
\begin{aligned}
& \mathcal{H}_{d_{1} \ldots d_{s}} \stackrel{\text { def }}{=} \hat{\mathcal{A}}_{d_{1} \ldots d_{s}} \hat{\mathcal{A}}_{d_{1} \ldots d_{s}}^{\dagger}+\tilde{\mathcal{E}}_{d_{s}}, \quad \mathcal{H}_{d_{1} \ldots d_{s}}=\left(\mathcal{H}_{d_{1} \ldots d_{s} x, y}\right) \quad\left(x, y=0,1, \ldots, x_{\max }\right), \\
& \hat{\mathcal{A}}_{d_{1} \ldots d_{s}} \stackrel{\text { def }}{=} \sqrt{\hat{B}_{d_{1} \ldots d_{s}}(x)}-e^{\partial} \sqrt{\hat{D}_{d_{1} \ldots d_{s}}(x)}, \quad \hat{\mathcal{A}}_{d_{1} \ldots d_{s}}^{\dagger}=\sqrt{\hat{B}_{d_{1} \ldots d_{s}}(x)}-\sqrt{\hat{D}_{d_{1} \ldots d_{s}}(x)} e^{-\partial}, \\
& \hat{B}_{d_{1} \ldots d_{s}}(x) \stackrel{\text { def }}{=} \alpha B^{\prime}(x+s-1) \frac{\mathrm{W}\left[\check{\xi}_{d_{1}}, \ldots, \check{\xi}_{d_{s-1}}\right](x)}{\mathrm{W}\left[\check{\xi}_{d_{1}}, \ldots, \check{\xi}_{d_{s-1}}\right](x+1)} \frac{\mathrm{W}\left[\check{\xi}_{d_{1}}, \ldots, \check{\xi}_{d_{s}}\right](x+1)}{\mathrm{W}\left[\check{\xi}_{d_{1}}, \ldots, \check{\xi}_{d_{s}}\right](x)} \\
& \hat{D}_{d_{1} \ldots d_{s}}(x) \stackrel{\text { def }}{=} \alpha D^{\prime}(x) \frac{\mathrm{W}\left[\check{\xi}_{d_{1}}, \ldots, \check{\xi}_{d_{s-1}}\right](x+1)}{\mathrm{W}\left[\check{\xi}_{d_{1}}, \ldots, \check{\xi}_{d_{s-1}}\right](x)} \frac{\mathrm{W}\left[\check{\xi}_{d_{1}}, \ldots, \check{\xi}_{d_{s}}\right](x-1)}{\mathrm{W}\left[\check{\xi}_{d_{1}}, \ldots, \check{\xi}_{d_{s}}\right](x)}
\end{aligned}
$$




$$
\begin{aligned}
& \phi_{d_{1} \ldots d_{s} n}(x) \stackrel{\text { def }}{=} \hat{\mathcal{A}}_{d_{1} \ldots d_{s}} \phi_{d_{1} \ldots d_{s-1} n}(x) \quad\left(x=0,1, \ldots, x_{\max } ; n=0,1, \ldots, n_{\max }\right) \\
& \tilde{\phi}_{d_{1} \ldots d_{s} \mathrm{v}}(x) \stackrel{\text { def }}{=} \hat{\mathcal{A}}_{d_{1} \ldots d_{s}} \tilde{\phi}_{d_{1} \ldots d_{s-1} \mathrm{v}}(x)+\delta_{x, x_{\max }} \varphi_{d_{1} \ldots d_{s} \mathrm{v}}, \quad\left(x=0,1, \ldots, x_{\max } ; \mathrm{v} \in \mathcal{V} \backslash\left\{d_{1}, \ldots, d_{s}\right\}\right) \\
& \varphi_{d_{1} \ldots d_{s} \mathrm{v}} \stackrel{\text { def }}{=} \phi_{d_{1} \ldots d_{s} 0}\left(x_{\max }\right) \frac{\mathrm{W}\left[\check{\xi}_{d_{1}}, \ldots, \check{\xi}_{d_{s}}\right]\left(x_{\max }\right) \mathrm{W}\left[\check{\xi}_{d_{1}}, \ldots, \check{\xi}_{d_{s-1}}, \check{\xi}_{\mathrm{v}}\right]\left(x_{\max }+1\right)}{\mathrm{W}\left[\check{\xi}_{d_{1}}, \ldots, \check{\xi}_{d_{s-1}}\right]\left(x_{\max }+1\right) \mathrm{W}\left[\check{\xi}_{d_{1}}, \ldots, \check{\xi}_{d_{s}}, \nu\right]\left(x_{\max }\right)} \\
& \mathcal{H}_{d_{1} \ldots d_{s}} \phi_{d_{1} \ldots d_{s} n}(x)=\mathcal{E}_{n} \phi_{d_{1} \ldots d_{s} n}(x) \quad\left(x=0,1, \ldots, x_{\max } ; n=0,1, \ldots, n_{\max }\right) \\
& \mathcal{H}_{d_{1} \ldots d_{s}} \tilde{\phi}_{d_{1} \ldots d_{s} \mathrm{v}}(x)=\tilde{\mathcal{E}}_{\mathrm{v}} \tilde{\phi}_{d_{1} \ldots d_{s} \mathrm{v}}(x) \quad\left(x=0,1, \ldots, x_{\max }-1 ; \mathrm{v} \in \mathcal{V} \backslash\left\{d_{1}, \ldots, d_{s}\right\}\right) \\
& \left(\phi_{d_{1} \ldots d_{s} n}, \phi_{d_{1} \ldots d_{s} m}\right) \stackrel{\text { def }}{=} \sum_{x=0}^{x_{\max }} \phi_{d_{1} \ldots d_{s} n}(x) \phi_{d_{1} \ldots d_{s} m}(x)=\prod_{j=1}^{s}\left(\mathcal{E}_{n}-\tilde{\mathcal{E}}_{d_{j}}\right) \cdot \frac{1}{d_{n}^{2}} \delta_{n m} \\
& \left(n, m=0,1, \ldots, n_{\max }\right)
\end{aligned}
$$

The eigenvectors and the virtual state vectors have Casoratian expressions $\left(x=0,1, \ldots, x_{\max }\right)$ :

$$
\begin{aligned}
\phi_{d_{1} \ldots d_{s} n}(x) & =\frac{(-1)^{s} \sqrt{\prod_{j=1}^{s} \alpha B^{\prime}(x+j-1)} \tilde{\phi}_{0}(x) \mathrm{W}\left[\check{\xi}_{d_{1}}, \ldots, \check{\xi}_{d_{s}}, \nu \check{P}_{n}\right](x)}{\sqrt{\mathrm{W}\left[\check{\xi}_{d_{1}}, \ldots, \check{\xi}_{d_{s}}\right](x) \mathrm{W}\left[\check{\xi}_{d_{1}}, \ldots, \check{\xi}_{d_{s}}\right](x+1)}}, \\
\tilde{\phi}_{d_{1} \ldots d_{s} \mathrm{v}}(x) & =\frac{(-1)^{s} \sqrt{\prod_{j=1}^{s} \alpha B^{\prime}(x+j-1)} \tilde{\phi}_{0}(x) \mathrm{W}\left[\check{\xi}_{d_{1}}, \ldots, \check{\xi}_{d_{s}}, \check{\xi}_{\mathrm{v}}\right](x)}{\sqrt{\mathrm{W}\left[\check{\xi}_{d_{1}}, \ldots, \check{\xi}_{d_{s}}\right](x) \mathrm{W}\left[\check{\xi}_{d_{1}}, \ldots, \check{\xi}_{d_{s}}\right](x+1)}} .
\end{aligned}
$$

The Casoratian in the virtual state vectors $\mathrm{W}\left[\check{\xi}_{d_{1}}, \ldots, \check{\xi}_{d_{s}}, \check{\xi}_{\mathrm{v}}\right](x)$ has definite sign for $x=$ $0,1, \ldots, x_{\max }+1$, and that appearing in the groundstate eigenvector $\mathrm{W}\left[\check{\xi}_{d_{1}}, \ldots, \check{\xi}_{d_{s}}, \nu\right](x)$ has definite sign for $x=0,1, \ldots, x_{\max }$, too.

The next step begins with rewriting the Hamiltonian $\mathcal{H}_{d_{1} \ldots d_{s}}$ by choosing the next virtual state to be deleted $d_{s+1} \in \mathcal{V} \backslash\left\{d_{1}, \ldots, d_{s}\right\}$. The potential functions $\hat{B}_{d_{1} \ldots d_{s+1}}(x)$ and $\hat{D}_{d_{1} \ldots d_{s+1}}(x)$ are defined as in $(\underline{3.13})$-(3.14) by $s \rightarrow s+1$. We have $\hat{B}_{d_{1} \ldots d_{s+1}}(x)>0(x=$ $\left.0,1, \ldots, x_{\max }\right), \hat{D}_{d_{1} \ldots d_{s+1}}(0)=\hat{D}_{d_{1} \ldots d_{s+1}}\left(x_{\max }+1\right)=0, \hat{D}_{d_{1} \ldots d_{s+1}}(x)>0\left(x=1,2, \ldots, x_{\max }\right)$. These functions satisfy the relations

$$
\begin{aligned}
& \hat{B}_{d_{1} \ldots d_{s+1}}(x) \hat{D}_{d_{1} \ldots d_{s+1}}(x+1)=\hat{B}_{d_{1} \ldots d_{s}}(x+1) \hat{D}_{d_{1} \ldots d_{s}}(x+1), \\
& \hat{B}_{d_{1} \ldots d_{s+1}}(x)+\hat{D}_{d_{1} \ldots d_{s+1}}(x)+\tilde{\mathcal{E}}_{d_{s+1}}=\hat{B}_{d_{1} \ldots d_{s}}(x)+\hat{D}_{d_{1} \ldots d_{s}}(x+1)+\tilde{\mathcal{E}}_{d_{s}} .
\end{aligned}
$$

The Hamiltonian $\mathcal{H}_{d_{1} \ldots d_{s}}$ is rewritten as:

$$
\begin{gathered}
\mathcal{H}_{d_{1} \ldots d_{s}}=\hat{\mathcal{A}}_{d_{1} \ldots d_{s+1}}^{\dagger} \hat{\mathcal{A}}_{d_{1} \ldots d_{s+1}}+\tilde{\mathcal{E}}_{d_{s+1}}, \\
\hat{\mathcal{A}}_{d_{1} \ldots d_{s+1}} \stackrel{\text { def }}{=} \sqrt{\hat{B}_{d_{1} \ldots d_{s+1}}(x)}-e^{\partial} \sqrt{\hat{D}_{d_{1} \ldots d_{s+1}}(x)}, \hat{\mathcal{A}}_{d_{1} \ldots d_{s+1}}^{\dagger}=\sqrt{\hat{B}_{d_{1} \ldots d_{s+1}}(x)}-\sqrt{\hat{D}_{d_{1} \ldots d_{s+1}}(x)} e^{-\partial} .
\end{gathered}
$$


Now let us define a new Hamiltonian $\mathcal{H}_{d_{1} \ldots d_{s+1}}$ by changing the orders of $\hat{\mathcal{A}}_{d_{1} \ldots d_{s+1}}^{\dagger}$ and $\hat{\mathcal{A}}_{d_{1} \ldots d_{s+1}}$ together with the eigenvectors $\phi_{d_{1} \ldots d_{s+1} n}(x)$ and the virtual state vectors $\tilde{\phi}_{d_{1} \ldots d_{s+1} \mathrm{v}}(x)$ :

$$
\begin{aligned}
& \mathcal{H}_{d_{1} \ldots d_{s+1}} \stackrel{\text { def }}{=} \hat{\mathcal{A}}_{d_{1} \ldots d_{s+1}} \hat{\mathcal{A}}_{d_{1} \ldots d_{s+1}}^{\dagger}+\tilde{\mathcal{E}}_{d_{s+1}}, \quad \mathcal{H}_{d_{1} \ldots d_{s+1}}=\left(\mathcal{H}_{d_{1} \ldots d_{s+1} x, y}\right) \quad\left(x, y=0,1, \ldots, x_{\max }\right), \\
& \phi_{d_{1} \ldots d_{s+1} n}(x) \stackrel{\text { def }}{=} \hat{\mathcal{A}}_{d_{1} \ldots d_{s+1}} \phi_{d_{1} \ldots d_{s} n}(x) \quad\left(x=0,1, \ldots, x_{\max } ; n=0,1, \ldots, n_{\max }\right), \\
& \tilde{\phi}_{d_{1} \ldots d_{s+1} \mathrm{v}}(x) \stackrel{\text { def }}{=} \hat{\mathcal{A}}_{d_{1} \ldots d_{s+1}} \tilde{\phi}_{d_{1} \ldots d_{s} \mathrm{v}}(x)+\delta_{x, x_{\max }} \varphi_{d_{1} \ldots d_{s+1} \mathrm{v}}, \\
& \qquad\left(x=0,1, \ldots, x_{\max } ; \mathrm{v} \in \mathcal{V} \backslash\left\{d_{1}, \ldots, d_{s+1}\right\}\right), \\
& \varphi_{d_{1} \ldots d_{s+1 \mathrm{v}} \mathrm{v}} \stackrel{\text { def }}{=} \phi_{d_{1} \ldots d_{s+1} 0}\left(x_{\max }\right) \frac{\mathrm{W}\left[\check{\xi}_{d_{1}}, \ldots, \check{\xi}_{d_{s+1}}\right]\left(x_{\max }\right) \mathrm{W}\left[\check{\xi}_{d_{1}}, \ldots, \check{\xi}_{d_{s}}, \check{\xi}_{\mathrm{v}}\right]\left(x_{\max }+1\right)}{\mathrm{W}\left[\check{\xi}_{d_{1}}, \ldots, \check{\xi}_{d_{s}}\right]\left(x_{\max }+1\right) \mathrm{W}\left[\check{\xi}_{d_{1}}, \ldots, \check{\xi}_{d_{s+1}}, \nu\right]\left(x_{\max }\right)} .
\end{aligned}
$$

The orthogonality relation reads

$$
\left(\phi_{d_{1} \ldots d_{s+1} n}, \phi_{d_{1} \ldots d_{s+1} m}\right)=\prod_{j=1}^{s+1}\left(\mathcal{E}_{n}-\tilde{\mathcal{E}}_{d_{j}}\right) \cdot \frac{1}{d_{n}^{2}} \delta_{n m} \quad\left(n, m=0,1, \ldots, n_{\max }\right) .
$$

The functions $\phi_{d_{1} \ldots d_{s+1} n}(x)$ and $\tilde{\phi}_{d_{1} \ldots d_{s+1} \mathrm{v}}(x)$ are expressed as Casoratians as in (3.20)(3.21). The Casoratian $\mathrm{W}\left[\check{\xi}_{d_{1}}, \ldots, \check{\xi}_{d_{s+1}}, \check{\xi}_{\mathrm{v}}\right](x)$ has definite sign

$$
\begin{aligned}
& \pm \frac{\mathrm{W}\left[\check{\xi}_{d_{1}}, \ldots, \check{\xi}_{d_{s}}\right](0)\left(\tilde{\mathcal{E}}_{d_{s+1}}-\tilde{\mathcal{E}}_{\mathrm{v}}\right)}{\mathrm{W}\left[\check{\xi}_{d_{1}}, \ldots, \check{\xi}_{d_{s+1}}\right](0) \mathrm{W}\left[\check{\xi}_{d_{1}}, \ldots, \check{\xi}_{d_{s}}, \check{\xi}_{\mathrm{v}}\right](0)}>0 \\
& \Rightarrow \pm \mathrm{W}\left[\check{\xi}_{d_{1}}, \ldots, \check{\xi}_{d_{s+1}}, \check{\xi}_{\mathrm{v}}\right](x)>0 \quad\left(x=0,1, \ldots, x_{\max }+1\right) .
\end{aligned}
$$

Likewise $\mathrm{W}\left[\check{\xi}_{d_{1}}, \ldots, \check{\xi}_{d_{s+1}}, \nu\right](x)$ and the lowest eigenvector $\phi_{d_{1} \ldots d_{s+1} 0}(x)$ have definite sign

$$
\begin{aligned}
& \mp \frac{\mathrm{W}\left[\check{\xi}_{d_{1}}, \ldots, \check{\xi}_{d_{s}}\right](0)}{\mathrm{W}\left[\check{\xi}_{d_{1}}, \ldots, \check{\xi}_{d_{s+1}}\right](0) \mathrm{W}\left[\check{\xi}_{d_{1}}, \ldots, \check{\xi}_{d_{s}}, \nu\right](0)}>0 \\
& \Rightarrow \pm \mathrm{W}\left[\check{\xi}_{d_{1}}, \ldots, \check{\xi}_{d_{s+1}}, \nu\right](x)>0 \quad\left(x=0,1, \ldots, x_{\max }\right) .
\end{aligned}
$$

These establish the $s+1$ case.

At the end of this subsection we present this deformed Hamiltonian $\mathcal{H}_{d_{1} \ldots d_{s}}$ in the standard form, in which the $\mathcal{A}$ operator annihilates the groundstate eigenvector:

$$
\begin{aligned}
& \mathcal{H}_{d_{1} \ldots d_{s}}=\mathcal{A}_{d_{1} \ldots d_{s}}^{\dagger} \mathcal{A}_{d_{1} \ldots d_{s}}, \\
& \mathcal{A}_{d_{1} \ldots d_{s}} \stackrel{\text { def }}{=} \sqrt{B_{d_{1} \ldots d_{s}}(x)}-e^{\partial} \sqrt{D_{d_{1} \ldots d_{s}}(x)}, \quad \mathcal{A}_{d_{1} \ldots d_{s}}^{\dagger}=\sqrt{B_{d_{1} \ldots d_{s}}(x)}-\sqrt{D_{d_{1} \ldots d_{s}}(x)} e^{-\partial},
\end{aligned}
$$

which satisfies

$$
\mathcal{A}_{d_{1} \ldots d_{s}} \phi_{d_{1} \ldots d_{s} 0}(x)=0 \quad\left(x=0,1, \ldots, x_{\max }\right) .
$$


The potential functions $B_{d_{1} \ldots d_{s}}(x)$ and $D_{d_{1} \ldots d_{s}}(x)$ are:

$$
\begin{aligned}
& B_{d_{1} \ldots d_{s}}(x) \stackrel{\text { def }}{=} \alpha B^{\prime}(x+s) \frac{\mathrm{W}\left[\check{\xi}_{d_{1}}, \ldots, \check{\xi}_{d_{s}}\right](x)}{\mathrm{W}\left[\check{\xi}_{d_{1}}, \ldots, \check{\xi}_{d_{s}}\right](x+1)} \frac{\mathrm{W}\left[\check{\xi}_{d_{1}}, \ldots, \check{\xi}_{d_{s}}, \nu\right](x+1)}{\mathrm{W}\left[\check{\xi}_{d_{1}}, \ldots, \check{\xi}_{d_{s}}, \nu\right](x)} \\
& D_{d_{1} \ldots d_{s}}(x) \stackrel{\text { def }}{=} \alpha D^{\prime}(x) \frac{\mathrm{W}\left[\check{\xi}_{d_{1}}, \ldots, \check{\xi}_{d_{s}}\right](x+1)}{\mathrm{W}\left[\check{\xi}_{d_{1}}, \ldots, \check{\xi}_{d_{s}}\right](x)} \frac{\mathrm{W}\left[\check{\xi}_{d_{1}}, \ldots, \check{\xi}_{d_{s}}, \nu\right](x-1)}{\mathrm{W}\left[\check{\xi}_{d_{1}}, \ldots, \check{\xi}_{d_{s}}, \nu\right](x)}
\end{aligned}
$$

The positivity of $B_{d_{1} \ldots d_{s}}(x)$ and $D_{d_{1} \ldots d_{s}}(x)$ is shown above and the boundary conditions $B_{d_{1} \ldots d_{s}}\left(x_{\max }\right)=0$ and $D_{d_{1} \ldots d_{s}}(0)=0$ are satisfied. They satisfy the relations

$$
\begin{aligned}
& B_{d_{1} \ldots d_{s}}(x) D_{d_{1} \ldots d_{s}}(x+1)=\hat{B}_{d_{1} \ldots d_{s}}(x+1) \hat{D}_{d_{1} \ldots d_{s}}(x+1) \\
& B_{d_{1} \ldots d_{s}}(x)+D_{d_{1} \ldots d_{s}}(x)=\hat{B}_{d_{1} \ldots d_{s}}(x)+\hat{D}_{d_{1} \ldots d_{s}}(x+1)+\tilde{\mathcal{E}}_{d_{s}} .
\end{aligned}
$$

It should be stressed that the above results after $s$-deletions are independent of the orders of deletions $\left(\phi_{d_{1} \ldots d_{s} n}(x)\right.$ and $\tilde{\phi}_{d_{1} \ldots d_{s} \mathrm{v}}(x)$ may change sign).

\subsection{Explicit forms of multi-indexed $(q-)$ Racah polynomials}

Here we present the main results of the paper. The multi-indexed $(q-)$ Racah polynomials are obtained by applying the method of virtual states deletion to the $(q-)$ Racah system. The parameter $\boldsymbol{\lambda}=\left(\lambda_{1}, \lambda_{2}, \ldots\right)$ dependence is now shown explicitly. The eigenvectors of the models in $\S 5$ of [2] are described by orthogonal polynomials in the sinusoidal coordinate $\eta(x ; \boldsymbol{\lambda})$. The auxiliary function $\varphi(x ; \boldsymbol{\lambda})$ is defined by

$$
\varphi(x ; \boldsymbol{\lambda}) \stackrel{\text { def }}{=} \frac{\eta(x+1 ; \boldsymbol{\lambda})-\eta(x ; \boldsymbol{\lambda})}{\eta(1 ; \boldsymbol{\lambda})},
$$

and it satisfies (with $\boldsymbol{\delta}$ defined in (2.15)-(2.16) $)$

$$
\frac{\varphi(x ; \boldsymbol{\lambda})}{\varphi(x-1 ; \boldsymbol{\lambda}+2 \boldsymbol{\delta})}=\varphi(1 ; \boldsymbol{\lambda}) .
$$

All the models in $\S 5$ of [2] have shape invariance [44]. The following relations are very useful:

$$
\begin{aligned}
& \varphi(x ; \boldsymbol{\lambda})=\sqrt{\frac{B(0 ; \boldsymbol{\lambda})}{B(x ; \boldsymbol{\lambda})}} \frac{\phi_{0}(x ; \boldsymbol{\lambda}+\boldsymbol{\delta})}{\phi_{0}(x ; \boldsymbol{\lambda})}, \quad \varphi(x ; \boldsymbol{\lambda})=\sqrt{\frac{B(0 ; \boldsymbol{\lambda})}{D(x+1 ; \boldsymbol{\lambda})}} \frac{\phi_{0}(x ; \boldsymbol{\lambda}+\boldsymbol{\delta})}{\phi_{0}(x+1 ; \boldsymbol{\lambda})}, \\
& \frac{B(x ; \boldsymbol{\lambda}+\boldsymbol{\delta})}{B(x+1 ; \boldsymbol{\lambda})}=\kappa^{-1} \frac{\varphi(x+1 ; \boldsymbol{\lambda})}{\varphi(x ; \boldsymbol{\lambda})}, \quad \frac{D(x ; \boldsymbol{\lambda}+\boldsymbol{\delta})}{D(x ; \boldsymbol{\lambda})}=\kappa^{-1} \frac{\varphi(x-1 ; \boldsymbol{\lambda})}{\varphi(x ; \boldsymbol{\lambda})} .
\end{aligned}
$$

We delete $M$ virtual state vectors labeled by

$$
\mathcal{D}=\left\{d_{1}, d_{2}, \ldots, d_{M}\right\} \quad\left(d_{j} \in \mathcal{V}: \text { mutually distinct }\right)
$$


and denote $\mathcal{H}_{d_{1} \ldots d_{M}}, \phi_{d_{1} \ldots d_{M} n}, \mathcal{A}_{d_{1} \ldots d_{M}}$, etc. by $\mathcal{H}_{\mathcal{D}}, \phi_{\mathcal{D} n}, \mathcal{A}_{\mathcal{D}}$, etc.

Let us denote the eigenvector $\phi_{\mathcal{D} n}(x ; \boldsymbol{\lambda})$ in (3.20) after $M$ deletions $(s=M)$ by $\phi_{\mathcal{D} n}^{\text {gen }}(x ; \boldsymbol{\lambda})$. We define two polynomials $\breve{\Xi}_{\mathcal{D}}(x ; \boldsymbol{\lambda})$ and $\check{P}_{\mathcal{D}, n}(x ; \boldsymbol{\lambda})$, to be called the denominator polynomial and the multi-indexed orthogonal polynomial, respectively, from the Casoratians as follows:

$$
\begin{aligned}
& \mathrm{W}\left[\check{\xi}_{d_{1}}, \ldots, \check{\xi}_{d_{M}}\right](x ; \boldsymbol{\lambda})=\mathcal{C}_{\mathcal{D}}(\boldsymbol{\lambda}) \varphi_{M}(x ; \boldsymbol{\lambda}) \check{\Xi}_{\mathcal{D}}(x ; \boldsymbol{\lambda}), \\
& \mathrm{W}\left[\check{\xi}_{d_{1}}, \ldots, \check{\xi}_{d_{M}}, \nu \check{P}_{n}\right](x ; \boldsymbol{\lambda})=\mathcal{C}_{\mathcal{D}, n}(\boldsymbol{\lambda}) \varphi_{M+1}(x ; \boldsymbol{\lambda}) \check{P}_{\mathcal{D}, n}(x ; \boldsymbol{\lambda}) \nu(x ; \boldsymbol{\lambda}+M \tilde{\boldsymbol{\delta}}), \\
& \tilde{\boldsymbol{\delta}} \stackrel{\text { def }}{=}(0,0,1,1), \quad \mathfrak{t}(\boldsymbol{\lambda})+\beta \boldsymbol{\delta}=\mathfrak{t}(\boldsymbol{\lambda}+\beta \tilde{\boldsymbol{\delta}}) \quad(\forall \beta \in \mathbb{R}) .
\end{aligned}
$$

The constants $\mathcal{C}_{\mathcal{D}}(\boldsymbol{\lambda})$ and $\mathcal{C}_{\mathcal{D}, n}(\boldsymbol{\lambda})$ are specified later. The auxiliary function $\varphi_{M}(x ; \boldsymbol{\lambda})$ is defined by [42]:

$$
\begin{aligned}
\varphi_{M}(x ; \boldsymbol{\lambda}) & \stackrel{\text { def }}{=} \prod_{1 \leq j<k \leq M} \frac{\eta(x+k-1 ; \boldsymbol{\lambda})-\eta(x+j-1 ; \boldsymbol{\lambda})}{\eta(k-j ; \boldsymbol{\lambda})} \\
& =\prod_{1 \leq j<k \leq M} \varphi(x+j-1 ; \boldsymbol{\lambda}+(k-j-1) \boldsymbol{\delta}),
\end{aligned}
$$

and $\varphi_{0}(x ; \boldsymbol{\lambda})=\varphi_{1}(x ; \boldsymbol{\lambda})=1$. The eigenvector (3.20) is rewritten as

$$
\begin{aligned}
\phi_{\mathcal{D} n}^{\mathrm{gen}}(x ; \boldsymbol{\lambda})= & (-1)^{M} \kappa^{\frac{1}{4} M(M-1)} \frac{\mathcal{C}_{\mathcal{D}, n}(\boldsymbol{\lambda})}{\mathcal{C}_{\mathcal{D}}(\boldsymbol{\lambda})} \sqrt{\prod_{j=1}^{M} \alpha(\boldsymbol{\lambda}) B^{\prime}(0 ; \boldsymbol{\lambda}+(j-1) \tilde{\boldsymbol{\delta}})} \\
& \times \frac{\phi_{0}(x ; \boldsymbol{\lambda}+M \tilde{\boldsymbol{\delta}})}{\sqrt{\check{\Xi}_{\mathcal{D}}(x ; \boldsymbol{\lambda}) \check{\Xi}_{\mathcal{D}}(x+1 ; \boldsymbol{\lambda})}} \check{P}_{\mathcal{D}, n}(x ; \boldsymbol{\lambda}) .
\end{aligned}
$$

The multi-indexed orthogonal polynomial $\check{P}_{\mathcal{D}, n}(x ; \boldsymbol{\lambda})(\underline{3.33})$ has an expression

$$
\begin{aligned}
\check{P}_{\mathcal{D}, n}(x ; \boldsymbol{\lambda})= & \mathcal{C}_{\mathcal{D}, n}(\boldsymbol{\lambda})^{-1} \varphi_{M+1}(x ; \boldsymbol{\lambda})^{-1} \\
& \times\left|\begin{array}{cccc}
\check{\xi}_{d_{1}}\left(x_{1}\right) & \cdots & \check{\xi}_{d_{M}}\left(x_{1}\right) & r_{1}\left(x_{1}\right) \check{P}_{n}\left(x_{1}\right) \\
\check{\xi}_{d_{1}}\left(x_{2}\right) & \cdots & \check{\xi}_{d_{M}}\left(x_{2}\right) & r_{2}\left(x_{2}\right) \check{P}_{n}\left(x_{2}\right) \\
\vdots & \cdots & \vdots & \vdots \\
\check{\xi}_{d_{1}}\left(x_{M+1}\right) & \cdots & \check{\xi}_{d_{M}}\left(x_{M+1}\right) & r_{M+1}\left(x_{M+1}\right) \check{P}_{n}\left(x_{M+1}\right)
\end{array}\right|,
\end{aligned}
$$

where $x_{j} \stackrel{\text { def }}{=} x+j-1$ and $r_{j}(x)=r_{j}(x ; \boldsymbol{\lambda}, M)(1 \leq j \leq M+1)$ are given by

$$
r_{j}(x+j-1 ; \boldsymbol{\lambda}, M) \stackrel{\text { def }}{=}\left\{\begin{array}{ll}
\frac{(x+a, x+b)_{j-1}(x+d-a+j, x+d-b+j)_{M+1-j}}{(d-a+1, d-b+1)_{M}} & : \mathrm{R} \\
\frac{\left(a q^{x}, b q^{x} ; q\right)_{j-1}\left(a^{-1} d q^{x+j}, b^{-1} d q^{x+j} ; q\right)_{M+1-j}}{\left(a b d^{-1} q^{-1}\right)^{j-1} q^{M x}\left(a^{-1} d q, b^{-1} d q ; q\right)_{M}} & : q \mathrm{R}
\end{array} .\right.
$$


One can show that $\check{\Xi}_{\mathcal{D}}(\underline{3.32})$ and $\check{P}_{\mathcal{D}, n}($ 3.37) are indeed polynomials in $\eta$ :

$$
\check{\Xi}_{\mathcal{D}}(x ; \boldsymbol{\lambda}) \stackrel{\text { def }}{=} \Xi_{\mathcal{D}}(\eta(x ; \boldsymbol{\lambda}+(M-1) \tilde{\boldsymbol{\delta}}) ; \boldsymbol{\lambda}), \quad \check{P}_{\mathcal{D}, n}(x ; \boldsymbol{\lambda}) \stackrel{\text { def }}{=} P_{\mathcal{D}, n}(\eta(x ; \boldsymbol{\lambda}+M \tilde{\boldsymbol{\delta}}) ; \boldsymbol{\lambda}),
$$

and their degrees are generically $\ell$ and $\ell+n$, respectively (see (3.59)). Here $\ell$ is

$$
\ell \stackrel{\text { def }}{=} \sum_{j=1}^{M} d_{j}-\frac{1}{2} M(M-1)
$$

The involution properties (2.31) of these polynomials are the consequence of those of the basic polynomials $\check{P}_{n}(x)$ and $\check{\xi}_{d_{j}}(x)$. We adopt the standard normalisation for $\check{\Xi}_{\mathcal{D}}$ and $\check{P}_{\mathcal{D}, n}$ : $\check{\Xi}_{\mathcal{D}}(0 ; \boldsymbol{\lambda})=1, \check{P}_{\mathcal{D}, n}(0 ; \boldsymbol{\lambda})=1$, which determine the constants $\mathcal{C}_{\mathcal{D}}(\boldsymbol{\lambda})$ and $\mathcal{C}_{\mathcal{D}, n}(\boldsymbol{\lambda})$,

$$
\begin{aligned}
\mathcal{C}_{\mathcal{D}}(\boldsymbol{\lambda}) \stackrel{\text { def }}{=} \frac{1}{\varphi_{M}(0 ; \boldsymbol{\lambda})} \prod_{1 \leq j<k \leq M} \frac{\tilde{\mathcal{E}}_{d_{j}}(\boldsymbol{\lambda})-\tilde{\mathcal{E}}_{d_{k}}(\boldsymbol{\lambda})}{\alpha(\boldsymbol{\lambda}) B^{\prime}(j-1 ; \boldsymbol{\lambda})} \\
\mathcal{C}_{\mathcal{D}, n}(\boldsymbol{\lambda}) \stackrel{\text { def }}{=}(-1)^{M} \mathcal{C}_{\mathcal{D}}(\boldsymbol{\lambda}) \tilde{d}_{\mathcal{D}, n}(\boldsymbol{\lambda})^{2}, \quad \tilde{d}_{\mathcal{D}, n}(\boldsymbol{\lambda})^{2} \stackrel{\text { def }}{=} \frac{\varphi_{M}(0 ; \boldsymbol{\lambda})}{\varphi_{M+1}(0 ; \boldsymbol{\lambda})} \prod_{j=1}^{M} \frac{\mathcal{E}_{n}(\boldsymbol{\lambda})-\tilde{\mathcal{E}}_{d_{j}}(\boldsymbol{\lambda})}{\alpha(\boldsymbol{\lambda}) B^{\prime}(j-1 ; \boldsymbol{\lambda})} .
\end{aligned}
$$

The use of dual polynomials $Q_{x}\left(\mathcal{E}_{n}\right) \stackrel{\text { def }}{=} P_{n}(\eta(x))$ [2] is essential for the derivation of these results. The three term recurrence relations of $\left\{Q_{x}(\mathcal{E})\right\}$ are specified by $B(x)$ and $D(x)$. The denominator polynomial $\check{\Xi}_{\mathcal{D}}(x ; \boldsymbol{\lambda})$ is positive for $x=0,1, \ldots, x_{\max }+1$. The lowest degree multi-indexed orthogonal polynomial $\check{P}_{\mathcal{D}, 0}(x ; \boldsymbol{\lambda})$ is related to $\check{\Xi}_{\mathcal{D}}(x ; \boldsymbol{\lambda})$ by the parameter shift $\lambda \rightarrow \boldsymbol{\lambda}+\boldsymbol{\delta}:$

$$
\check{P}_{\mathcal{D}, 0}(x ; \boldsymbol{\lambda})=\check{\Xi}_{\mathcal{D}}(x ; \boldsymbol{\lambda}+\boldsymbol{\delta}) .
$$

The potential functions $B_{\mathcal{D}}$ and $D_{\mathcal{D}}(3.25)-(3.26)$ after $M$-deletion $(s=M)$ can be expressed neatly in terms of the denominator polynomial:

$$
\begin{aligned}
& B_{\mathcal{D}}(x ; \boldsymbol{\lambda})=B(x ; \boldsymbol{\lambda}+M \tilde{\boldsymbol{\delta}}) \frac{\check{\Xi}_{\mathcal{D}}(x ; \boldsymbol{\lambda})}{\check{\Xi}_{\mathcal{D}}(x+1 ; \boldsymbol{\lambda})} \frac{\check{\Xi}_{\mathcal{D}}(x+1 ; \boldsymbol{\lambda}+\boldsymbol{\delta})}{\check{\Xi}_{\mathcal{D}}(x ; \boldsymbol{\lambda}+\boldsymbol{\delta})}, \\
& D_{\mathcal{D}}(x ; \boldsymbol{\lambda})=D(x ; \boldsymbol{\lambda}+M \tilde{\boldsymbol{\delta}}) \frac{\check{\Xi}_{\mathcal{D}}(x+1 ; \boldsymbol{\lambda})}{\check{\Xi}_{\mathcal{D}}(x ; \boldsymbol{\lambda})} \frac{\check{\Xi}_{\mathcal{D}}(x ; \boldsymbol{\lambda}+\boldsymbol{\delta})}{\check{\Xi}_{\mathcal{D}}(x ; \boldsymbol{\lambda}+\boldsymbol{\delta})} .
\end{aligned}
$$

These formulas look similar to those in the exceptional polynomials [23]. The groundstate eigenvector $\phi_{\mathcal{D} 0}$ is expressed by $\phi_{0}(x)(2.8)$ and $\check{\Xi}_{\mathcal{D}}(x ; \boldsymbol{\lambda})$ :

$$
\phi_{\mathcal{D} 0}(x ; \boldsymbol{\lambda})=\sqrt{\prod_{y=0}^{x-1} \frac{B_{\mathcal{D}}(y)}{D_{\mathcal{D}}(y+1)}}=\phi_{0}(x ; \boldsymbol{\lambda}+M \tilde{\boldsymbol{\delta}}) \sqrt{\frac{\check{\Xi}_{\mathcal{D}}(1 ; \boldsymbol{\lambda})}{\check{\Xi}_{\mathcal{D}}(x ; \boldsymbol{\lambda}) \check{\Xi}_{\mathcal{D}}(x+1 ; \boldsymbol{\lambda})}} \check{\Xi}_{\mathcal{D}}(x ; \boldsymbol{\lambda}+\boldsymbol{\delta})
$$




$$
\begin{gathered}
=\psi_{\mathcal{D}}(x ; \boldsymbol{\lambda}) \check{P}_{\mathcal{D}, 0}(x ; \boldsymbol{\lambda}) \propto \phi_{\mathcal{D} 0}^{\text {gen }}(x ; \boldsymbol{\lambda}), \\
\psi_{\mathcal{D}}(x ; \boldsymbol{\lambda}) \stackrel{\text { def }}{=} \sqrt{\check{\Xi}_{\mathcal{D}}(1 ; \boldsymbol{\lambda})} \frac{\phi_{0}(x ; \boldsymbol{\lambda}+M \tilde{\boldsymbol{\delta}})}{\sqrt{\check{\Xi}_{\mathcal{D}}(x ; \boldsymbol{\lambda}) \check{\Xi}_{\mathcal{D}}(x+1 ; \boldsymbol{\lambda})}}, \quad \psi_{\mathcal{D}}(0 ; \boldsymbol{\lambda})=1 .
\end{gathered}
$$

We arrive at the normalised eigenvector $\phi_{\mathcal{D} n}(x ; \boldsymbol{\lambda})$ with the orthogonality relation,

$$
\begin{aligned}
& \phi_{\mathcal{D} n}(x ; \boldsymbol{\lambda}) \stackrel{\text { def }}{=} \psi_{\mathcal{D}}(x ; \boldsymbol{\lambda}) \check{P}_{\mathcal{D}, n}(x ; \boldsymbol{\lambda}) \propto \phi_{\mathcal{D} n}^{\text {gen }}(x ; \boldsymbol{\lambda}), \quad \phi_{\mathcal{D} n}(0 ; \boldsymbol{\lambda})=1, \\
& \sum_{x=0}^{x_{\max }} \frac{\psi_{\mathcal{D}}(x ; \boldsymbol{\lambda})^{2}}{\check{\Xi}_{\mathcal{D}}(1 ; \boldsymbol{\lambda})} \check{P}_{\mathcal{D}, n}(x ; \boldsymbol{\lambda}) \check{P}_{\mathcal{D}, m}(x ; \boldsymbol{\lambda})=\frac{\delta_{n m}}{d_{n}(\boldsymbol{\lambda})^{2} \tilde{d}_{\mathcal{D}, n}(\boldsymbol{\lambda})^{2}} \quad\left(n, m=0,1, \ldots, n_{\max }\right) .
\end{aligned}
$$

It is worthwhile to emphasise that the above orthogonality relation is a rational equation of $\boldsymbol{\lambda}$ or $q^{\boldsymbol{\lambda}}$, and it is valid for any value of $\boldsymbol{\lambda}$ (except for the zeros of denominators) but the weight function may not be positive definite.

The shape invariance of the original system is inherited by the deformed systems. The matrix $\hat{\mathcal{A}}_{d_{1} \ldots d_{s+1}}(\boldsymbol{\lambda})$ intertwines the two Hamiltonians $\mathcal{H}_{d_{1} \ldots d_{s}}(\boldsymbol{\lambda})$ and $\mathcal{H}_{d_{1} \ldots d_{s+1}}(\boldsymbol{\lambda})$,

$$
\begin{aligned}
& \hat{\mathcal{A}}_{d_{1} \ldots d_{s+1}}(\boldsymbol{\lambda})^{\dagger} \hat{\mathcal{A}}_{d_{1} \ldots d_{s+1}}(\boldsymbol{\lambda})=\mathcal{H}_{d_{1} \ldots d_{s}}(\boldsymbol{\lambda})-\tilde{\mathcal{E}}_{d_{s+1}}(\boldsymbol{\lambda}), \\
& \hat{\mathcal{A}}_{d_{1} \ldots d_{s+1}}(\boldsymbol{\lambda}) \hat{\mathcal{A}}_{d_{1} \ldots d_{s+1}}(\boldsymbol{\lambda})^{\dagger}=\mathcal{H}_{d_{1} \ldots d_{s+1}}(\boldsymbol{\lambda})-\tilde{\mathcal{E}}_{d_{s+1}}(\boldsymbol{\lambda}),
\end{aligned}
$$

and it has the inverse. By the same argument given in $\S 4$ of [19], the shape invariance of $\mathcal{H}(\boldsymbol{\lambda})$ is inherited by $\mathcal{H}_{d_{1}}(\boldsymbol{\lambda}), \mathcal{H}_{d_{1} d_{2}}(\boldsymbol{\lambda}), \cdots$. Therefore the Hamiltonian $\mathcal{H}_{\mathcal{D}}(\boldsymbol{\lambda})$ is shape invariant:

$$
\mathcal{A}_{\mathcal{D}}(\boldsymbol{\lambda}) \mathcal{A}_{\mathcal{D}}(\boldsymbol{\lambda})^{\dagger}=\kappa \mathcal{A}_{\mathcal{D}}(\boldsymbol{\lambda}+\boldsymbol{\delta})^{\dagger} \mathcal{A}_{\mathcal{D}}(\boldsymbol{\lambda}+\boldsymbol{\delta})+\mathcal{E}_{1}(\boldsymbol{\lambda})
$$

As a consequence of the shape invariance and the normalisation, the actions of $\mathcal{A}_{\mathcal{D}}(\boldsymbol{\lambda})$ and $\mathcal{A}_{\mathcal{D}}(\boldsymbol{\lambda})^{\dagger}$ on the eigenvectors $\phi_{\mathcal{D} n}(x ; \boldsymbol{\lambda})$ are

$$
\begin{aligned}
& \mathcal{A}_{\mathcal{D}}(\boldsymbol{\lambda}) \phi_{\mathcal{D} n}(x ; \boldsymbol{\lambda})=\frac{\mathcal{E}_{n}(\boldsymbol{\lambda})}{\sqrt{B_{\mathcal{D}}(0 ; \boldsymbol{\lambda})}} \phi_{\mathcal{D} n-1}(x ; \boldsymbol{\lambda}+\boldsymbol{\delta}) \quad\left(x=0,1, \ldots, x_{\max }-1\right), \\
& \mathcal{A}_{\mathcal{D}}(\boldsymbol{\lambda})^{\dagger} \phi_{\mathcal{D} n-1}(x ; \boldsymbol{\lambda}+\boldsymbol{\delta})=\sqrt{B_{\mathcal{D}}(0 ; \boldsymbol{\lambda})} \phi_{\mathcal{D} n}(x ; \boldsymbol{\lambda}) \quad\left(x=0,1, \ldots, x_{\max }\right) .
\end{aligned}
$$

The forward and backward shift operators are defined by

$$
\begin{aligned}
\mathcal{F}_{\mathcal{D}}(\boldsymbol{\lambda}) & \stackrel{\text { def }}{=} \sqrt{B_{\mathcal{D}}(0 ; \boldsymbol{\lambda})} \psi_{\mathcal{D}}(x ; \boldsymbol{\lambda}+\boldsymbol{\delta})^{-1} \circ \mathcal{A}_{\mathcal{D}}(\boldsymbol{\lambda}) \circ \psi_{\mathcal{D}}(x ; \boldsymbol{\lambda}) \\
& =\frac{B(0 ; \boldsymbol{\lambda}+M \tilde{\boldsymbol{\delta}})}{\varphi(x ; \boldsymbol{\lambda}+M \tilde{\boldsymbol{\delta}}) \check{\Xi}_{\mathcal{D}}(x+1 ; \boldsymbol{\lambda})}\left(\check{\Xi}_{\mathcal{D}}(x+1 ; \boldsymbol{\lambda}+\boldsymbol{\delta})-\check{\Xi}_{\mathcal{D}}(x ; \boldsymbol{\lambda}+\boldsymbol{\delta}) e^{\partial}\right), \\
\mathcal{B}_{\mathcal{D}}(\boldsymbol{\lambda}) & \stackrel{\text { def }}{=} \frac{1}{\sqrt{B_{\mathcal{D}}(0 ; \boldsymbol{\lambda})}} \psi_{\mathcal{D}}(x ; \boldsymbol{\lambda})^{-1} \circ \mathcal{A}_{\mathcal{D}}(\boldsymbol{\lambda})^{\dagger} \circ \psi_{\mathcal{D}}(x ; \boldsymbol{\lambda}+\boldsymbol{\delta})
\end{aligned}
$$




$$
\begin{aligned}
= & \frac{1}{B(0 ; \boldsymbol{\lambda}+M \tilde{\boldsymbol{\delta}}) \check{\Xi}_{\mathcal{D}}(x ; \boldsymbol{\lambda}+\boldsymbol{\delta})} \\
& \times\left(B(x ; \boldsymbol{\lambda}+M \tilde{\boldsymbol{\delta}}) \check{\Xi}_{\mathcal{D}}(x ; \boldsymbol{\lambda})-D(x ; \boldsymbol{\lambda}+M \tilde{\boldsymbol{\delta}}) \check{\Xi}_{\mathcal{D}}(x+1 ; \boldsymbol{\lambda}) e^{-\partial}\right) \varphi(x ; \boldsymbol{\lambda}+M \tilde{\boldsymbol{\delta}}),
\end{aligned}
$$

and their actions on $\check{P}_{\mathcal{D}, n}(x ; \boldsymbol{\lambda})$ are

$$
\mathcal{F}_{\mathcal{D}}(\boldsymbol{\lambda}) \check{P}_{\mathcal{D}, n}(x ; \boldsymbol{\lambda})=\mathcal{E}_{n}(\boldsymbol{\lambda}) \check{P}_{\mathcal{D}, n-1}(x ; \boldsymbol{\lambda}+\boldsymbol{\delta}), \quad \mathcal{B}_{\mathcal{D}}(\boldsymbol{\lambda}) \check{P}_{\mathcal{D}, n-1}(x ; \boldsymbol{\lambda}+\boldsymbol{\delta})=\check{P}_{\mathcal{D}, n}(x ; \boldsymbol{\lambda})
$$

As in the original (q-) Racah theory (2.33), these formulas are useful for the explicit calculation of the multi-indexed polynomials. The similarity transformed Hamiltonian is

$$
\begin{aligned}
& \widetilde{\mathcal{H}}_{\mathcal{D}}(\boldsymbol{\lambda}) \stackrel{\text { def }}{=} \psi_{\mathcal{D}}(x ; \boldsymbol{\lambda})^{-1} \circ \mathcal{H}_{\mathcal{D}}(\boldsymbol{\lambda}) \circ \psi_{\mathcal{D}}(x ; \boldsymbol{\lambda})=\mathcal{B}_{\mathcal{D}}(\boldsymbol{\lambda}) \mathcal{F}_{\mathcal{D}}(\boldsymbol{\lambda}) \\
&=B(x ; \boldsymbol{\lambda}+M \tilde{\boldsymbol{\delta}}) \frac{\check{\Xi}_{\mathcal{D}}(x ; \boldsymbol{\lambda})}{\check{\Xi}_{\mathcal{D}}(x+1 ; \boldsymbol{\lambda})}\left(\frac{\check{\Xi}_{\mathcal{D}}(x+1 ; \boldsymbol{\lambda}+\boldsymbol{\delta})}{\check{\Xi}_{\mathcal{D}}(x ; \boldsymbol{\lambda}+\boldsymbol{\delta})}-e^{\partial}\right) \\
&+D(x ; \boldsymbol{\lambda}+M \tilde{\boldsymbol{\delta}}) \frac{\check{\Xi}_{\mathcal{D}}(x+1 ; \boldsymbol{\lambda})}{\check{\Xi}_{\mathcal{D}}(x ; \boldsymbol{\lambda})}\left(\frac{\check{\Xi}_{\mathcal{D}}(x-1 ; \boldsymbol{\lambda}+\boldsymbol{\delta})}{\check{\Xi}_{\mathcal{D}}(x ; \boldsymbol{\lambda}+\boldsymbol{\delta})}-e^{-\partial}\right),
\end{aligned}
$$

and the multi-indexed orthogonal polynomials $\check{P}_{\mathcal{D}, n}(x ; \boldsymbol{\lambda})$ are its eigenpolynomials:

$$
\widetilde{\mathcal{H}}_{\mathcal{D}}(\boldsymbol{\lambda}) \check{P}_{\mathcal{D}, n}(x ; \boldsymbol{\lambda})=\mathcal{E}_{n}(\boldsymbol{\lambda}) \check{P}_{\mathcal{D}, n}(x ; \boldsymbol{\lambda})
$$

Other intertwining relations are

$$
\begin{aligned}
\kappa^{\frac{1}{2}} \hat{\mathcal{A}}_{d_{1} \ldots d_{s+1}}(\boldsymbol{\lambda}+\boldsymbol{\delta}) \mathcal{A}_{d_{1} \ldots d_{s}}(\boldsymbol{\lambda}) & =\mathcal{A}_{d_{1} \ldots d_{s+1}}(\boldsymbol{\lambda}) \hat{\mathcal{A}}_{d_{1} \ldots d_{s+1}}(\boldsymbol{\lambda}) \\
\kappa^{-\frac{1}{2}} \hat{\mathcal{A}}_{d_{1} \ldots d_{s+1}}(\boldsymbol{\lambda}) \mathcal{A}_{d_{1} \ldots d_{s}}(\boldsymbol{\lambda})^{\dagger} & =\mathcal{A}_{d_{1} \ldots d_{s+1}}(\boldsymbol{\lambda})^{\dagger} \hat{\mathcal{A}}_{d_{1} \ldots d_{s+1}}(\boldsymbol{\lambda}+\boldsymbol{\delta})
\end{aligned}
$$

with the potential functions given in (3.13)-(3.14) (with $s \rightarrow s+1$ ).

Including the level 0 deletion corresponds to $M-1$ virtual states deletion:

$$
\left.\check{P}_{\mathcal{D}, n}(x ; \boldsymbol{\lambda})\right|_{d_{M}=0}=\check{P}_{\mathcal{D}^{\prime}, n}(x ; \boldsymbol{\lambda}+\tilde{\boldsymbol{\delta}}), \quad \mathcal{D}^{\prime}=\left\{d_{1}-1, \ldots, d_{M-1}-1\right\}
$$

This formula is similar to those in the multi-indexed Jacobi theory, eqs.(48)-(49) in [1]. The denominator polynomial $\Xi_{\mathcal{D}}$ behaves similarly. This is why we have restricted $d_{j} \geq 1$.

The coefficients of the highest degree terms of the polynomials $\Xi_{\mathcal{D}}$ and $P_{\mathcal{D}, n}$,

$$
\begin{aligned}
\Xi_{\mathcal{D}}(y ; \boldsymbol{\lambda}) & =c_{\mathcal{D}}^{\Xi}(\boldsymbol{\lambda}) y^{\ell}+(\text { lower order terms }), \\
P_{\mathcal{D}, n}(y ; \boldsymbol{\lambda}) & =c_{\mathcal{D}, n}^{P}(\boldsymbol{\lambda}) y^{\ell+n}+(\text { lower order terms }),
\end{aligned}
$$


are

$$
\begin{aligned}
& c_{\mathcal{D}}^{\Xi}(\boldsymbol{\lambda})= \begin{cases}\frac{\prod_{j=1}^{M}\left(-a-b+c+d+d_{j}+1\right)_{d_{j}}}{\prod_{1 \leq j<k \leq M}\left(-a-b+c+d+d_{j}+d_{k}+1\right)} \prod_{j=1}^{M} \frac{(c, d-a+1, d-b+1)_{j-1}}{(c, d-a+1, d-b+1)_{d_{j}}} & : \mathrm{R} \\
\frac{\prod_{j=1}^{M}\left(a^{-1} b^{-1} c d q^{d_{j}+1} ; q\right)_{d_{j}}}{\prod_{1 \leq j<k \leq M}\left(1-a^{-1} b^{-1} c d q^{d_{j}+d_{k}+1}\right)} \prod_{j=1}^{M} \frac{\left(c, a^{-1} d q, b^{-1} d q ; q\right)_{j-1}}{\left(c, a^{-1} d q, b^{-1} d q ; q\right)_{d_{j}}} & : q \mathrm{R}\end{cases} \\
& c_{\mathcal{D}, n}^{P}(\boldsymbol{\lambda})=c_{\mathcal{D}}^{\Xi}(\boldsymbol{\lambda}) \times \begin{cases}\frac{(a+b+c-d+n-1)_{n}(c)_{M}}{(a, b, c)_{n} \prod_{j=1}^{M}\left(c+n+d_{j}\right)} & : \mathrm{R} \\
\frac{\left(a b c d^{-1} q^{n-1} ; q\right)_{n}(c ; q)_{M}}{(a, b, c ; q)_{n} \prod_{j=1}^{M}\left(1-c q^{\left.n+d_{j}\right)}\right.} & : q \mathrm{R}\end{cases}
\end{aligned}
$$

The exceptional $X_{\ell}\left(q^{-}\right)$Racah orthogonal polynomials presented in [23] correspond to the simplest case $M=1, \mathcal{D}=\{\ell\}, \ell \geq 1$ :

$$
\check{\xi}_{\ell}(x ; \boldsymbol{\lambda})=\check{\Xi}_{\{\ell\}}(x ; \boldsymbol{\lambda}+\ell \boldsymbol{\delta}-\tilde{\boldsymbol{\delta}}), \quad \check{P}_{\ell, n}(x ; \boldsymbol{\lambda})=\check{P}_{\{\ell\}, n}(x ; \boldsymbol{\lambda}+\ell \boldsymbol{\delta}-\tilde{\boldsymbol{\delta}}) .
$$

\section{Summary and Comments}

Following the examples of multi-indexed Laguerre and Jacobi polynomials [1], multi-indexed $(q-)$ Racah polynomials, the discrete quantum mechanics counterparts, are constructed. These new polynomials could be considered as a further generalisation of Bannai-Ito polynomials [45]. The next stage will be the construction of multi-indexed Askey-Wilson and Wilson polynomials. The basic logic is the same for the ordinary quantum mechanics as well as for the discrete quantum mechanics with real [2] or pure imaginary shifts [28]. Starting from the factorised Hamiltonians of exactly solvable quantum mechanical systems, a series of new 'deformed' exactly solvable quantum systems are generated by applying Crum-Krein-Adler formulas [36, 37] or multiple Darboux transformations [39] through deletion of various virtual states instead of eigenstates. The virtual state vectors are polynomial 'solutions' of a virtual Hamiltonian which is obtained by twisting the discrete symmetry of the original Hamiltonian. They fail to satisfy the Schrödinger equation of the virtual Hamiltonian at one of the boundaries, at $x=x_{\max }$. When there is only one extra index $\mathcal{D}=\{\ell\}(\ell \geq 1)$, the multi-indexed $(q-)$ Racah polynomials reduce to the exceptional polynomials [23, 26]. Like the exceptional polynomials, the multi-indexed $(q-)$ Racah polynomials do not satisfy the three term recurrence relations. On the other hand, their dual polynomials satisfy the three term recurrence relations because of the tri-diagonal form of the Hamiltonian. As for the 
parameter ranges in which (2.42)-(2.43), (2.53)-(2.54) are satisfied, we have taken conservative ones, (2.18)-(2.19), (2.39), (2.55). It is quite possible that the valid parameter ranges could be enlarged. The difference equations for the multi-indexed $(q-)$ Racah polynomials, (3.55), (3.57) are purely algebraic and they hold for any parameter ranges.

As in the ordinary Sturm-Liouville case (the oscillation theorem) the multi-indexed orthogonal polynomial $P_{\mathcal{D}, n}(y ; \boldsymbol{\lambda})$ has $n$ zeros in the orthogonality range, $0<y<\eta\left(x_{\max } ; \boldsymbol{\lambda}+\right.$ $M \tilde{\boldsymbol{\delta}})$. This is a general property of the eigenvectors of a Jacobi matrix of the form (2.1), (2.45) etc. See [46].

A few words on the mirror reflection with respect to the mid point of the $x$-grid, $x \rightarrow$ $x_{\max }-x=N-x$. The original $(q-)$ Racah polynomial under the reflection is described by the same polynomial with different parameters:

$$
\begin{gathered}
\check{P}_{n}(N-x ; \boldsymbol{\lambda})=A \times \check{P}_{n}\left(x ;\left(\lambda_{1}, \lambda_{1}+\lambda_{3}-\lambda_{4}, \lambda_{1}+\lambda_{2}-\lambda_{4}, 2 \lambda_{1}-\lambda_{4}\right)\right), \\
A=\check{P}_{n}(N ; \boldsymbol{\lambda})=\left\{\begin{array}{cc}
\frac{(a+b-d, a+c-d)_{n}}{(b, c)_{n}} & : \mathrm{R} \\
\left(a^{-1} d\right)^{n} \frac{\left(a b d^{-1}, a c d^{-1} ; q\right)_{n}}{(b, c ; q)_{n}} & : q \mathrm{R}
\end{array}\right.
\end{gathered}
$$

This corresponds to the mirror reflection formula of the Jacobi polynomials:

$$
P_{n}^{(\alpha, \beta)}(-\eta)=(-1)^{n} P_{n}^{(\beta, \alpha)}(\eta), \quad \eta(x)=\cos 2 x, \quad \eta\left(\frac{\pi}{2}-x\right)=-\eta(x) .
$$

The type I and II virtual state wavefunctions for the Jacobi case have a twisted boundary condition at $x=\frac{\pi}{2}$ and $x=0$, respectively [1] and their polynomial parts are related by this mirror reflection [14]. The virtual state vectors, which fail to satisfy the equation at $x=x_{\max }$, correspond to the type I. By the mirror reflection, one can consider the type II virtual state vectors, which fail to satisfy the equation at $x=0$, instead of $x=x_{\max }$. By using these type II virtual state vectors, the mirror reflexed version of the multi-indexed $(q-)$ Racah polynomials can be constructed. They are related to the multi-indexed $(q-)$ Racah polynomials by

$$
\begin{aligned}
\check{P}_{\mathcal{D}, n}^{\text {mirror }}(x ; \boldsymbol{\lambda}) & =A \times \check{P}_{\mathcal{D}, n}\left(N-x ;\left(\lambda_{1}, \lambda_{1}+\lambda_{3}-\lambda_{4}, \lambda_{1}+\lambda_{2}-\lambda_{4}, 2 \lambda_{1}-\lambda_{4}\right)\right), \\
A^{-1} & =\check{P}_{\mathcal{D}, n}\left(N ;\left(\lambda_{1}, \lambda_{1}+\lambda_{3}-\lambda_{4}, \lambda_{1}+\lambda_{2}-\lambda_{4}, 2 \lambda_{1}-\lambda_{4}\right)\right) .
\end{aligned}
$$

The normalisation conditions are $\check{P}_{n}(0 ; \boldsymbol{\lambda})=\check{P}_{\mathcal{D}, n}(0 ; \boldsymbol{\lambda})=\check{P}_{\mathcal{D}, n}^{\text {mirror }}(0 ; \boldsymbol{\lambda})=1$. Contrary to the Jacobi case [1], the type I and II virtual state vectors cannot be used together to generate new multi-indexed $(q-)$ Racah polynomials. 
Various orthogonal polynomials are obtained from the $(q-)$ Racah polynomials in certain limits. Similarly, from the multi-indexed $(q-)$ Racah polynomials presented in the previous section, we can obtain the multi-indexed version of various orthogonal polynomials, such as the (q-)Hahn, dual ( $q$-)Hahn, alternative $q$-Hahn (see $\S$ V.C.1 of [2]), etc. The infinite dimensional cases, the little $q$-Jacobi, $(q-)$ Meixner, etc will be reported in a separate publication. In certain limiting processes, the mirror reflection does not commute with the limit, as is well known in the Jacobi $\rightarrow$ Laguerre limits [14, 1]. The mirror reflexed multi-indexed polynomials are supposed to play certain roles in such limits.

Let us emphasise that the discrete symmetries of the original (q-)Racah systems and their twisting, which are essential for the construction of virtual Hamiltonians and virtual state vectors, are easily recognised in the present parametrisation (2.20)-(2.21) [2], but rather unclear in the original parametrisation [3, 4, 5]. This is a good reason to promote the $(q-)$ Racah systems in our parametrisation.

\section{Acknowledgements}

We thank a referee for many useful comments. S. O. thanks to his late father Zen Odake for warm encouragement. R. S. is supported in part by Grant-in-Aid for Scientific Research from the Ministry of Education, Culture, Sports, Science and Technology (MEXT), No.23540303 and No.22540186.

\section{References}

[1] S. Odake and R. Sasaki, "Exactly Solvable Quantum Mechanics and Infinite Families of Multi-indexed Orthogonal Polynomials," Phys. Lett. B702 (2011) 164-170, arXiv: 1105.0508 [math-ph].

[2] S. Odake and R. Sasaki, "Orthogonal Polynomials from Hermitian Matrices," J. Math. Phys. 49 (2008) 053503 (43pp), arXiv:0712.4106[math.CA]. (The dual q-Meixner polynomial in $§ 5.2 .4$ and dual $q$-Charlier polynomial in $\S 5.2 .8$ should be deleted because the hermiticity of the Hamiltonian is lost for these two cases.)

[3] G. E. Andrews, R. Askey and R. Roy, Special Functions, vol. 71 of Encyclopedia of mathematics and its applications, Cambridge Univ. Press, Cambridge, (1999). 
[4] M. E. H. Ismail, Classical and quantum orthogonal polynomials in one variable, vol. 98 of Encyclopedia of mathematics and its applications, Cambridge Univ. Press, Cambridge, (2005).

[5] R. Koekoek and R.F. Swarttouw, "The Askey-scheme of hypergeometric orthogonal polynomials and its q-analogue," arXiv:math.CA/9602214.

[6] G. Gasper and M. Rahman, Basic Hypergeometric Series (2nd ed.), vol. 96 of Encyclopedia of mathematics and its applications, Cambridge Univ. Press, Cambridge, (2004).

[7] A. F. Nikiforov, S. K. Suslov, and V.B. Uvarov, Classical Orthogonal Polynomials of a Discrete Variable, Springer, Berlin, (1991).

[8] D. Gómez-Ullate, N. Kamran and R. Milson, "An extension of Bochner's problem: exceptional invariant subspaces," J. Approx Theory 162 (2010) 987-1006, arXiv:0805. 3376 [math-ph]; "An extended class of orthogonal polynomials defined by a SturmLiouville problem," J. Math. Anal. Appl. 359 (2009) 352-367, arXiv:0807.3939 [math-ph].

[9] C. Quesne, "Exceptional orthogonal polynomials, exactly solvable potentials and supersymmetry," J. Phys. A41 (2008) 392001, arXiv:0807.4087[quant-ph]; B. Bagchi, C. Quesne and R. Roychoudhury, "Isospectrality of conventional and new extended potentials, second-order supersymmetry and role of PT symmetry," Pramana J. Phys. 73 (2009) 337-347, arXiv:0812.1488[quant-ph] .

[10] S. Odake and R. Sasaki, "Infinitely many shape invariant potentials and new orthogonal polynomials," Phys. Lett. B679 (2009) 414-417, arXiv:0906.0142[math-ph].

[11] C. Quesne, "Solvable rational potentials and exceptional orthogonal polynomials in supersymmetric quantum mechanics," SIGMA 5 (2009) 084, arXiv:0906.2331[math$\mathrm{ph}$.

[12] S. Odake and R. Sasaki, "Infinitely many shape invariant discrete quantum mechanical systems and new exceptional orthogonal polynomials related to the Wilson and AskeyWilson polynomials," Phys. Lett. B682 (2009) 130-136, arXiv:0909.3668[math-ph]. 
[13] S. Odake and R. Sasaki, "Infinitely many shape invariant potentials and cubic identities of the Laguerre and Jacobi polynomials," J. Math. Phys. 51 (2010) 053513 (9pp), arXiv:0911.1585[math-ph].

[14] S. Odake and R. Sasaki, "Another set of infinitely many exceptional $\left(X_{\ell}\right)$ Laguerre polynomials," Phys. Lett. B684 (2010) 173-176, arXiv:0911.3442[math-ph]. (Remark: $\mathrm{J} 1(\mathrm{~J} 2)$ in this reference corresponds to $\mathrm{J} 2(\mathrm{~J} 1)$ in later references.)

[15] B. Midya and B. Roy, "Exceptional orthogonal polynomials and exactly solvable potentials in position dependent mass Schrödinger Hamiltonians," Phys. Lett. A 373 (2009) 4117-4122.

[16] C.-L. Ho, S. Odake and R.Sasaki, "Properties of the exceptional $\left(X_{\ell}\right)$ Laguerre and Jacobi polynomials," SIGMA 7 (2011) 107 (24pp), arXiv:0912.5447[math-ph].

[17] D. Gómez-Ullate, N. Kamran and R. Milson, "Exceptional orthogonal polynomials and the Darboux transformation," J. Phys. A43 (2010) 434016 (16pp), arXiv:1002.2666 [math-ph]; "On orthogonal polynomials spanning a nonstandard flag," Contemp. Math. 563 (2012) 51-70, arXiv: 1101.5584 [math-ph].

[18] R. Sasaki, S. Tsujimoto and A. Zhedanov, "Exceptional Laguerre and Jacobi polynomials and the corresponding potentials through Darboux-Crum transformations," J. Phys. A43 (2010) 315204 (20pp), arXiv:1004.4711[math-ph].

[19] S. Odake and R. Sasaki, "Exceptional Askey-Wilson type polynomials through DarbouxCrum transformations," J. Phys. A43 (2010) 335201 (18pp), arXiv:1004.0544][math$\mathrm{ph}$.

[20] S. Odake and R. Sasaki, "A new family of shape invariantly deformed Darboux-PöschlTeller potentials with continuous $\ell$," J. Phys. A 44 (2011) 195203 (14pp), arXiv: 1007. $3800[\mathrm{math}-\mathrm{ph}]$.

[21] C-L.Ho, "Dirac(-Pauli), Fokker-Planck equations and exceptional Laguerre polynomials," Ann. Phys. 326 (2011) 797-807, arXiv:1008.0744[quant-ph].

[22] C-L. Ho and R.Sasaki, "Zeros of the exceptional Laguerre and Jacobi polynomials," ISRN Mathematical Physics, in press, arXiv:1102.5669[math-ph]. 
[23] S. Odake and R. Sasaki, "Exceptional $\left(X_{\ell}\right)(q-)$ Racah polynomials," Prog. Theor. Phys. 125 (2011) 851-870, arXiv:1102.0813[math-ph].

[24] Y. Grandati, "Solvable rational extensions of the isotonic oscillator," Ann. Phys. 326 (2011) 2074-2090, arXiv:1101.0055[math-ph]; "Solvable rational extensions of the Morse and Kepler-Coulomb potentials," arXiv:1103.5023[math-ph] .

[25] C-L. Ho, "Prepotential approach to solvable rational potentials and exceptional orthogonal polynomials," Prog. Theor. Phys. 126 (2011) 185-201,arXiv:1104.3511[math-ph].

[26] S. Odake and R. Sasaki, "Discrete quantum mechanics," J. Phys. A: Math. Theor. 44 (2011) 353001 (47pp), arXiv:1104.0473[math-ph].

[27] C. Quesne, "Higher-order SUSY, exactly solvable potentials, and exceptional orthogonal polynomials," Mod. Phys. Lett. A 26 (2011) 1843-1852, arXiv:1106.1990[math-ph]; "Rationally-extended radial oscillators and Laguerre exceptional orthogonal polynomials in kth-order SUSYQM," J. Mod. Phys. A 26 (2011) 5337-5347, arXiv:1110.3958 [math-ph]; "Exceptional orthogonal polynomials and new exactly solvable potentials in quantum mechanics," arXiv:1111.6467[math-ph].

[28] S. Odake and R. Sasaki, "Exactly solvable 'discrete' quantum mechanics; shape invariance, Heisenberg solutions, annihilation-creation operators and coherent states," Prog. Theor. Phys. 119 (2008) 663-700, arXiv:0802.1075[quant-ph].

[29] S. Odake and R. Sasaki, "Unified theory of exactly and quasi-exactly solvable 'discrete' quantum mechanics: I. Formalism," J. Math. Phys 51 (2010) 083502 (24pp). arXiv: 0903. $2604[$ math-ph].

[30] E. Routh, "On some properties of certain solutions of a differential equation of the second order," Proc. London Math. Soc. 16 (1884) 245-261; S. Bochner, "Über SturmLiouvillesche Polynomsysteme," Math. Zeit. 29 (1929) 730-736.

[31] D. Gómez-Ullate, N. Kamran and R. Milson, "Two-step Darboux transformations and exceptional Laguerre polynomials," J. Math. Anal. Appr. 387 (2012) 410-418, arXiv: 1103.5724[math-ph]. 
[32] P. R. Parthasarathy and R. B. Lenin, "Birth and death processes (BDP) models with applications," American Sciences Press, Inc. Columbus, Ohio (2004).

[33] R. Sasaki, "Exactly solvable birth and death processes," J. Math. Phys. 50 (2009) 103509 (18pp), arXiv:0903.3097[math-ph].

[34] S. Karlin and J. L. McGregor, "The differential equations of birth-and-death processes," Trans. Amer. Math. Soc. 85 (1957) 489-546.

[35] C. Albanese, M. Christandl, N. Datta and A. Ekert, "Mirror Inversion of Quantum States in Linear Registers," Phys. Rev. Lett. 93 (2004) 230502 (4pp), arXiv:quant-ph/0405 029; R. Chakrabarti and J.Van der Jeugt, "Quantum communication through a spin chain with interaction determined by a Jacobi matrix," J. Phys. A43 (2010) 085302 (20pp), arXiv:0912.0837[quant-ph]; L. Vinet and A. Zhedanov, "How to construct spin chains with perfect state transfer," arXiv:1110.6474[quant-ph].

[36] M. M. Crum, "Associated Sturm-Liouville systems," Quart. J. Math. Oxford Ser. (2) 6 (1955) 121-127, arXiv:physics/9908019.

[37] M. G. Krein, "On continuous analogue of a formula of Christoffel from the theory of orthogonal polynomials," Doklady Acad. Nauk. CCCP, 113 (1957) 970-973; V.É. Adler, "A modification of Crum's method," Theor. Math. Phys. 101 (1994) 1381-1386.

[38] A. A. Andrianov, M. V. Ioffe and V.P.Spiridonov, "Higher-derivative supersymmetry and the Witten index," Phys. Lett. A 174 (1993) 273-279; H. Aoyama, M. Sato and T. Tanaka, "General forms of a $\mathcal{N}$-fold supersymmetric family," Phys. Lett. B 503 (2001) 423-429, arXiv:quant-ph/0012065; D. J. Fernández and C. V. Hussin, "Higherorder SUSY, linearized nonlinear Heisenberg algebras and coherent states," J. Phys. A 32 (1999) 3603-3619; V. G. Bagrov and B. F. Samsonov, "Supersymmetry of a nonstationary Schrödinger equation," Phys. Lett. A 210 (1996) 60-64.

[39] G. Darboux, Théorie générale des surfaces vol 2 (1888) Gauthier-Villars, Paris.

[40] S. Odake and R. Sasaki, "Crum's theorem for 'discrete' quantum mechanics," Prog. Theor. Phys. 122 (2009) 1067-1079, arXiv:0902.2593[math-ph]. 
[41] L. García-Gutiérrez, S. Odake and R. Sasaki, "Modification of Crum's Theorem for 'Discrete' Quantum Mechanics," Prog. Theor. Phys. 124 (2010) 1-26, arXiv:1004.0289 [math-ph].

[42] S. Odake and R. Sasaki, "Dual Christoffel transformations," Prog. Theor. Phys. 126 (2011) 1-34, arXiv:1101.5468[math-ph].

[43] S. Odake and R. Sasaki, "Unified theory of annihilation-creation operators for solvable ('discrete') quantum mechanics," J. Math. Phys. 47 (2006) 102102 (33pp), arXiv: quant-ph/0605215; "Exact solution in the Heisenberg picture and annihilation-creation operators," Phys. Lett. B641 (2006) 112-117, arXiv:quant-ph/0605221.

[44] L. E. Gendenshtein, "Derivation of exact spectra of the Schroedinger equation by means of supersymmetry," JETP Lett. 38 (1983) 356-359.

[45] E. Bannai and T. Ito, Algebraic Combinatorics I: Association Schemes, Benjamin/Cummings, Menlo Park, CA, (1984).

[46] G. M. L. Gladwell, Inverse Problems in Vibration, Kluwer, Dordrecht (2004). 